\title{
On the use of a $\mathbf{P M}_{2.5}$ exposure simulator to explain birthweight
}

\author{
Veronica J. Berrocal ${ }^{a *}$, Alan E. Gelfand ${ }^{b}$, David M. Holland ${ }^{\mathrm{c}}$, Janet Burke \\ and Marie Lynn Miranda ${ }^{\mathrm{d}}$
}

\begin{abstract}
In relating pollution to birth outcomes, maternal exposure has usually been described using monitoring data. Such characterization provides a misrepresentation of exposure as (i) it does not take into account the spatial misalignment between an individual's residence and monitoring sites, and (ii) it ignores the fact that individuals spend most of their time indoors and typically in more than one location. In this paper, we break with previous studies by using a stochastic simulator to describe personal exposure (to particulate matter) and then relate simulated exposures at the individual level to the health outcome (birthweight) rather than aggregating to a selected spatial unit. We propose a hierarchical model that, at the first stage, specifies a linear relationship between birthweight and personal exposure, adjusting for individual risk factors and introduces random spatial effects for the census tract of maternal residence. At the second stage, we specify the distribution of each individual's personal exposure using the empirical distribution yielded by the stochastic simulator as well as a model for the spatial random effects. We have applied our framework to analyze birthweight data from 14 counties in North Carolina in years 2001 and 2002. We investigate whether there are certain aspects and time windows of exposure that are more detrimental to birthweight by building different exposure metrics which we incorporate, one by one, in our hierarchical model. To assess the difference in relating ambient exposure (i.e., exposure to ambient concentration) to birthweight versus personal exposure to birthweight, we compare estimates of the effect of air pollution obtained from hierarchical models that linearly relate ambient exposure and birthweight to those obtained from our modeling framework. Our analysis does not show a significant effect of $\mathbf{P M}_{2.5}$ on birthweight for reasons which we discuss. However, our modeling framework serves as a template for general analyses of the relationship between personal exposure and longer term health endpoints. Copyright (C) 2011 John Wiley \& Sons, Ltd.
\end{abstract}

Keywords: conditionally autoregressive (CAR) model; exposure metrics; hierarchical model; stochastic simulator

\section{INTRODUCTION}

The contribution of this paper is to develop a prototype hierarchical model for assessing the effect of air pollution on a long-term health outcome such as birthweight. We use a stochastic human exposure simulator, captured through empirical distributions, driven by ambient exposure obtained through fusing monitoring data and atmospheric numerical model output. We model at the individual level rather than aggregating to counts at census units in order to incorporate individual level risk factors. However, spatial random effects are introduced at the census unit level. We offer novel exposure metrics beyond average concentration which can be created due to the high spatial and temporal resolution in our pollution data. We propagate uncertainty in exposure through the hierarchical model to obtain appropriate uncertainty in the effect of exposure as well as in predictions using the model.

Air pollution has an adverse effect on human health: increasing concentration of pollutants are associated with a significant increase in nonaccidental mortality (Goldberg et al., 2001), total mortality (Dockery et al., 1993; Schwartz, 1994; Katsouyanni et al., 2001; Fuentes et al., 2006), cardiovascular disease (Braga et al., 2001; Hoek et al., 2001), respiratory deaths (Ostro et al., 2000; Braga et al., 2001), and morbity in general (Pope et al., 1995; Schwartz, 1999; Samet et al., 2000; Zanobetti et al., 2000). Health risks are not homogeneously spread across the population: elderly (Saldiva et al., 1995; Katsouyanni et al., 2001), children (Dockery and Pope, 1994; Schwartz et al., 1994; Heinrich et al., 1999), and infants (Bobak and Leon, 1992; Woodruff et al., 1997) are more vulnerable to the harmful effects of air pollution than the general adult population.

\footnotetext{
* Correspondence to: Veronica J. Berrocal, Assistant Professor, Department of Biostatistics, School of Public Health, University of Michigan, 1420 Washington Heights, Ann Arbor, MI 48109, USA.E-mail: berrocal@umich.edu

a Department of Biostatistics, University of Michigan, Ann Arbor, MI, USA

b Department of Statistical Science, Duke University, Durham, NC, USA

c U.S. Environmental Protection Agency, National Exposure Research Laboratory, Research Triangle Park, NC, USA

d The Nicholas School of the Environment, Duke University, Durham, NC, USA
} 
More recently, it has been suggested that fetuses might also be susceptible to the noxious effects of air pollution and a growing body of research on air pollution and birth outcomes has been carried out to validate such hypothesis. Studies have been conducted in several parts of the United States as well as around the world. In the United States, researchers have examined the relationship between air pollution and birth outcomes in California (Ritz and Yu, 1999; Ritz et al., 2000; Basu et al., 2004; Parker et al., 2005), Nevada (Chen et al., 2002), Georgia (Rogers and Dunlop, 2000, 2005), Connecticut and Massachusets (Bell et al., 2007), Northeastern US (Maisonet et al., 2001), and the entire US (Woodruff et al., 1997). Internationally, studies have been conducted in Brazil (Gouveia et al., 2004), Canada (Liu et al., 2003; Dugandzic et al., 2006), Korea (Ha et al., 2001; Lee et al., 2003), Taiwan (Yang et al., 2003; Lin et al., 2004), Australia (Mannes et al., 2005; Hansen et al., 2006), China (Wang et al., 1997), and the Czech Republic (Bobak, 2000). In each of these studies, the effects of exposure to several pollutants (ozone, particulate matter, total suspended particles, sulphur dioxide, nitrogen dioxide, and carbon monoxide) during different phases of the pregnancy (first trimester, last trimester, entire pregnancy) on birth outcomes (birth weight, low birth weight, preterm delivery, intrauterine growth restrictions and retardation, birth defects) have been examined with various results. Some authors have found an increase in the risk of low birth weight or premature birth as mothers are exposed to increasing concentrations of either pollutants during the entire pregnancy or earlier/later stages of the pregnancy. Others did not find a significant association between exposure and birth outcomes during any particular time window. Reviews on some of the literature on the effect of air pollution on pregnancy outcomes can be found in Šrám (1999), Maisonet et al. (2004) and Šrám et al. (2005).

Despite differences in spatial locations, populations considered, temporal windows of exposure and/or pollutants examined, all the studies on air pollution and birth outcomes share a similar characteristic. Maternal exposure to a pollutant is usually quantified by taking the average concentration, over the selected time window, of the pollutant under consideration as it is reported by outdoor monitors. While using monitoring data might seem a natural choice, there are two main problems with such a characterization of exposure: (i) monitors are not located where subjects live and (ii) individuals do not spend all of their time outdoors. As a consequence, ambient exposure, that is, exposure to an air pollutant in an outdoor environment, should not be considered equivalent to personal exposure and care should be taken in extrapolating the relationship between ambient exposure and birth outcomes to personal exposure and birth outcomes.

During the course of a day, an individual is exposed to pollution generated from sources that are located both outdoors and indoors. Therefore an individual's daily personal exposure is the sum of the exposure to pollutants accumulated in these two macro-environments. It is clear then that personal exposure and ambient exposure are not equal (Dockery and Spengler, 1981; Spengler et al., 1985; Lioy et al., 1990), and studies have also shown that they are only weakly correlated (Lioy et al., 1990; Özkaynak et al., 1996; Wallace, 1996). In light of this, using ambient exposure as a proxy for personal exposure in health studies might lead to biases both in the estimate of the effect of air pollution (Armstrong et al., 1992; Zeger et al., 2000) and in the assessment of its uncertainty (Samet et al., 2000; Gryparis et al.).

To account for the discrepancy between ambient and personal exposure, Dominici et al. (2000) propose a hierarchical measurement error model that links personal exposure to mortality in Baltimore in two stages. In the first stage, Dominici et al. (2000) use external data from five different cities to estimate the relationship between average ambient concentration and average personal exposure. They use this relationship to derive personal exposures for Baltimore, given the ambient concentration. Then, they establish a linear relationship between the derived personal exposures and mortality. Using a similar framework and considering again the city of Baltimore, McBride et al. (2007) presents a Bayesian hierarchical model whose aim is to characterize the relationship between personal exposure to fine particulate matter, that is $\mathrm{PM}_{2.5}$, and ambient concentration.

In both of these studies, personal exposure is derived from ambient exposure using a regression model. A different approach to obtain personal exposures is by using exposure simulators. Exposure simulators are stochastic models that predict the average personal exposure to a pollutant for demographic subgroups by randomly sampling individuals from each demographic group and randomly associating to each individual a time activity pattern that matches the subject in terms of personal characteristics, day of the week, temperature, season, etc. A description of the theoretical probabilistic framework underlying exposure simulators such as pNEM (Law et al., 1997), SHEDS-PM (Burke et al., 2001), APEX (Ozone 2006) and pCNEM (Zidek et al., 2003, 2007) can be found in Zidek et al. (2003).

Initially, exposure simulators were developed with the goal of determining the effect of ambient regulatory strategies on human exposure by observing how different ambient concentration scenarios changed the predicted personal exposure for each demographic group. Recently, they have been used as a tool to estimate personal exposures that are subsequently incorporated into statistical models relating air pollution to health outcomes. Holloman et al. (2004) and Calder et al. (2008) use a deterministic simplified version of the SHEDS-PM simulator to derive personal exposures to $\mathrm{PM}_{2.5}$ for eight counties in North Carolina for which they analyze mortality data. Shaddick et al. (2008) obtain sets of personal exposures to $\mathrm{PM}_{10}$ for eight districts in the Greater London area using the pCNEM simulator. A parametric distribution is fitted to these exposures which provides the second stage specification in a Bayesian hierarchical model that relates exposure to mortality counts for senior citizens. A similar approach is employed by Reich et al. (2009), who estimate personal exposures using the SHEDS-PM simulator and relate them to mortality in Fresno (CA) using a Bayesian hierarchical model. In this work, as in the others mentioned above, both the health outcome and the personal exposure are not modeled at the individual level, but rather they are aggregated over an area.

As noted at the outset, we present a methodological template for employing contaminant exposure simulators to explain a health outcome when both the personal exposure and the health outcome are considered at the individual level. Again, this enables us to introduce individual level risk factors. In particular, we consider $\mathrm{PM}_{2.5}$ as the pollutant, birthweight as the health outcome and we use the SHEDS-PM model (Burke et al., 2001) as the exposure simulator. SHEDS-PM enables us to account for variation in the daily activity of pregnant women and hence to capture uncertainty in individual exposure.

To assess the difference in relating ambient exposure and birthweight versus relating personal exposure and birthweight, we compare the use of ambient exposure developed through fusion of monitoring station data and computer model output with exposure developed through the simulator. This allows us to address a long-standing question of whether more reliable measures of exposure provide a different signal with regard to effect on birthweight outcomes. Additionally, to characterize maternal exposure over the course of a pregnancy we have built several metrics that take us beyond average concentration and also allow us to investigate various windows of vulnerability. We introduce 
these, one at time, into a regression model to explain birthweight. The simulator provides us only with empirical prior distributions for these metrics, not actual realizations for a given pregnancy. The uncertainty in these exposure metrics is then propagated to uncertainty in birthweight via a Bayesian hierarchical model that we fit through MCMC methods.

For the dataset we work with, we find a weak story regardless of how we introduce exposure; there seems to be no evidence of a relationship between $\mathrm{PM}_{2.5}$ exposure and birthweight. We discuss why, for our analysis, this might be expected. Still, taking a broader view, our approach can be viewed as a prototype for future investigations of this sort.

The format of the paper is as follows. In Section 2 we describe the birth outcome dataset we used as well as exposure data. In Section 3 we describe the SHEDS-PM model and how we used it here. Section 4 develops the various metrics we use to characterize exposure during a pregnancy. Section 5 develops the hierarchical model we employ while Section 6 summarizes the results of our analyses. We conclude with a summary and discussion in Section 7.

\section{DATA}

\subsection{Birth outcome data}

The Detailed Birth Record (DBR) database documents every live birth occurring in the state of North Carolina since 1990. For each birth, the DBR database reports the weight, originally measured in pounds (0-14) and ounces (0-16) and subsequently transformed in grams, the plurality, the number of children born dead and alive, the sex, the presence/absence of congenital anomalies and/or maternal complications, and the gestation length, clinically estimated and measured in weeks. The database also contains extensive information on maternal and paternal demographics, such as maternal and paternal race, maternal education, maternal age, maternal tobacco and alcohol use, marital status, and maternal residence. For our analysis, we have restricted our attention to birth outcome data for years 2001 and 2002 since these are the years for which $\mathrm{PM}_{2.5}$ concentration data was available to us. Since our interest is to study the relationship between air quality and birth outcomes, in this study we only consider births occurring in the counties in the corridor along the two major interstate highways that cross the center of the state, Interstate 85 and Interstate 40, where we would expect the air quality to be the poorest in the state. Those are also the counties that include the major urban areas and population centers in North Carolina. The 14 North Carolina counties and associated census tracts included in this study are shown in Figure 6 in the Appendix. They are, from South to North: Gaston, Mecklenburg, Cabarrus, Rowan, Davidson, Randolph, Guilford, Alamance, Orange, Durham, Granville, and Vance. We note that, though these 14 counties provide the highest $\mathrm{PM}_{2.5}$ exposures in North Carolina, they are moderate compared to levels observed in previous studies linking such exposure to health outcomes.

As our goal is to determine the effect on birthweight of maternal exposure to $\mathrm{PM}_{2.5}$ over the course of the pregnancy, we have only analyzed birth outcomes relative to pregnancies for which the entire gestation is fully included in the period January 1, 2001-December 31, 2002. We have also restricted the birth outcome data based on personal characteristics of the mother. Precisely, we have only considered birth outcomes for mothers who self-described themselves as either "Non-Hispanic White", "Non-Hispanic Black" or "Hispanics", with an age between 15 and 44 years, who had a gestation of at least 24 weeks and at most 42 weeks, and for which information on maternal tobacco and alcohol use was available. Finally, we excluded from our study births that were less than 400 grams, births with congenital anomalies, and non-singleton births.

In total, our dataset comprises 49869 births. Of these, the majority, 59.4\%, were from non-hispanic white mothers, while $25.7 \%$ were from non-hispanic black mothers and $15.0 \%$ from hispanic mothers. Maternal education was reported as number of years of schools completed. We subsequently grouped this variable in five categories: "middle school", "some high school", "high school", "some college," and "college". About $35 \%$ of the mothers in our dataset graduated from college, while $20.4 \%$ went through some years of it but did not complete it. Among the remaining mothers, $24.1 \%$ graduated from high school, $14.1 \%$ did not complete it and only $6.7 \%$ has a middle school diploma. Maternal age was originally recorded in integer years; however, to facilitate the analysis and the merging of SHEDS-PM simulated individuals with mothers in our birth outcomes dataset, we grouped maternal age into four age classes: 15-17 years, 18-24, 25-34, and $35-44$. About $54 \%$ of the births in our dataset were from mothers aged between 25 and 34 years, while only $3.4 \%$ were from mothers aged between 15 and 17 years of age. We have also included in our analysis marital status (32.5\% are married) and self-reported maternal tobacco use during pregnancy $(9.5 \%)$.

Finally, with the use of the Geographical Information System (GIS) software, it has been possible to geocode every birth in the dataset to the census tract of residence of the mother. This has enabled us to analyze the birth outcome data spatially, as the model in Section 5 indicates. A spatial map of the average birthweight for each census tract considered in this study is presented in Figure 1(a). As the figure shows, the average birthweight in the central counties of Alamance, Orange, and Durham is among the largest. However, the same counties are also characterized by great variability in birthweight (figure not shown).

\subsection{Air pollution data}

Particulate matter (PM) consists of small particles of solids and liquids that are suspended in the air. They are introduced in the atmosphere as a result of both biological and chemical processes and human activities (motor vehicles, power plants and wood burning, for example). In our study, we focus only on $\mathrm{PM}_{2.5}$ (also called fine particulate matter) and we examine the effect of exposure to fine particulate matter on birth outcomes. Our air pollution data refers to daily concentration of $\mathrm{PM}_{2.5}$. Two different types of data on $\mathrm{PM}_{2.5}$ concentration were available to us: monitoring data collected at sites sparsely located over North Carolina and gridded predictions of daily $\mathrm{PM}_{2.5}$ average concentration. The latter were produced by the Models-3/Community Multiscale Air Quality (CMAQ; http:// www.epa.gove/asmdnerl/CMAQ), a numerical model that estimates daily concentration of $\mathrm{PM}_{2.5}$ by integrating information coming from three different components: a meteorological component which accounts for the state and evolution in time of the atmosphere, an 


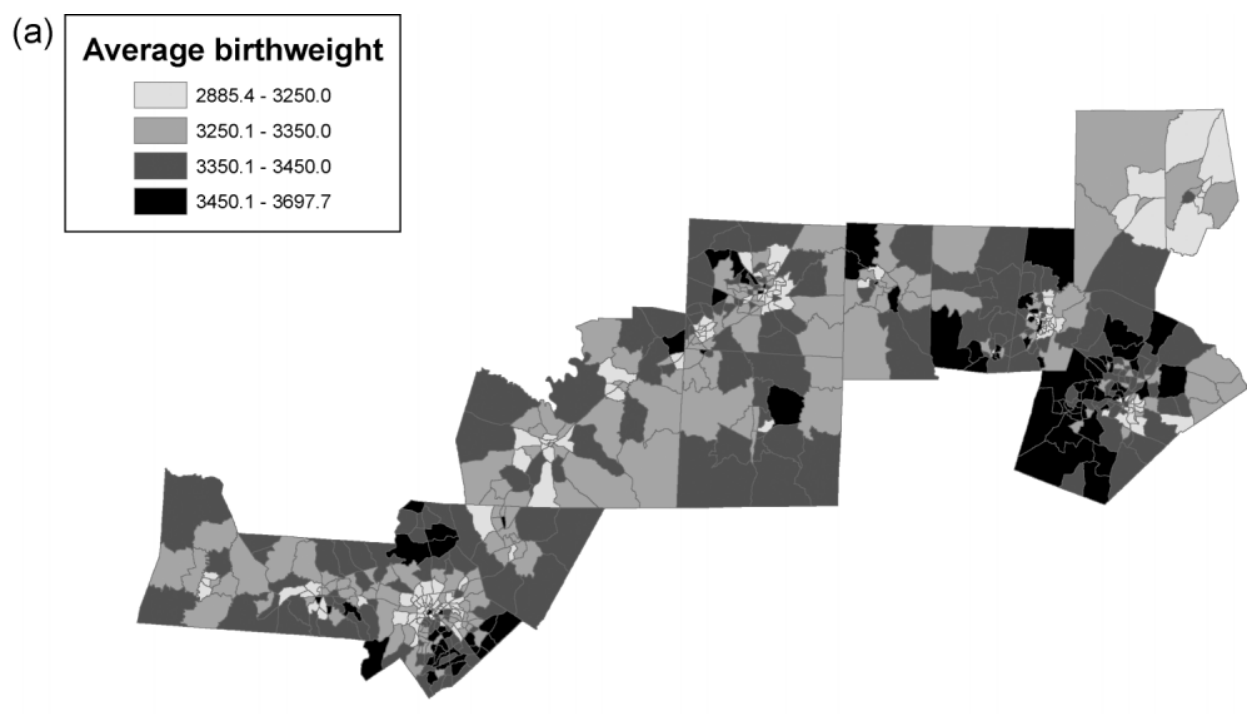

(b)

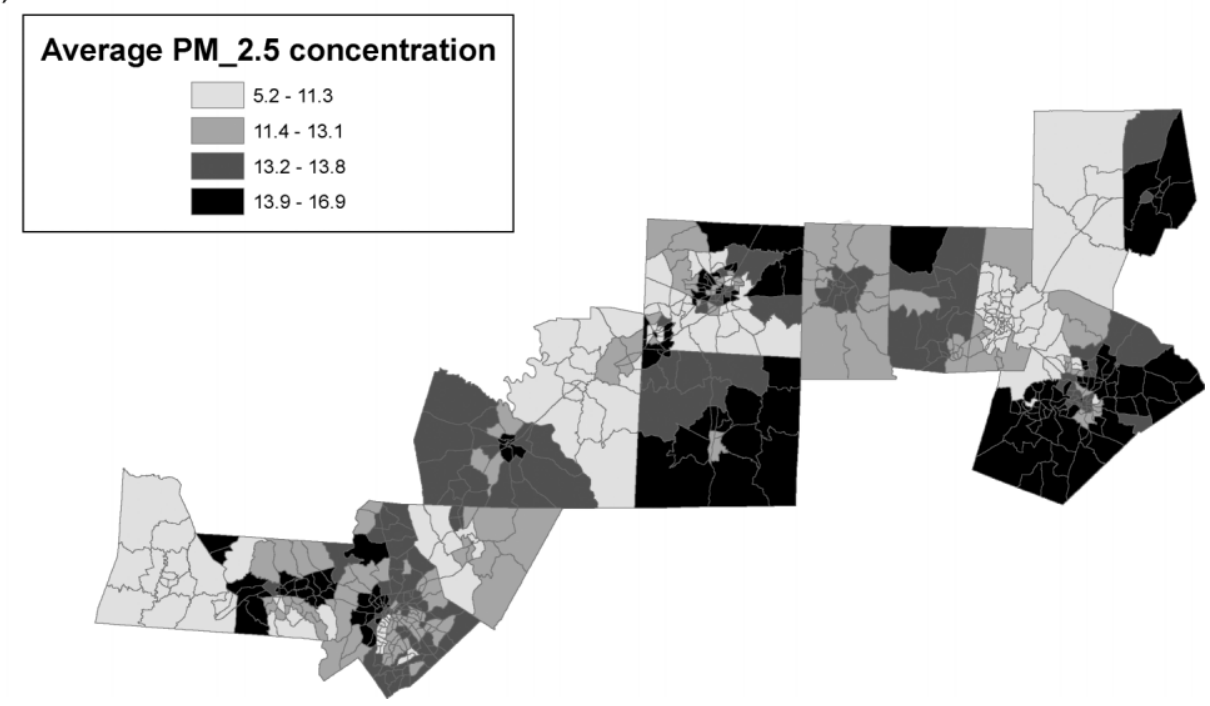

Figure 1. (a) Average birthweight and (b) average $\mathrm{PM}_{2.5}$ concentration (in $\mu \mathrm{g} / \mathrm{m}^{3}$ ) by census tract in 14 counties in North Carolina during years $2001-2002$. In both panels the breakpoints are the quartiles of the variable displayed

emission component which deals with emission injected in the atmosphere by both chemical plants and natural processes, and a component that accounts for the chemical and physical interactions occurring in the atmosphere. For our study we have used daily predictions of $\mathrm{PM}_{2.5}$ concentration obtained from the CMAQ numerical model run at the 12-km spatial resolution. The CMAQ output is available for each day and at a higher spatial resolution than the monitoring network though it is known to have calibration issues. The monitoring station data is not necessarily collected daily but, when available, it provides measurements that would be deemed more reliable than CMAQ output.

We use the fusion approach of McMillan et al. (2009) to exploit both sources of information (monitoring data and CMAQ output). Hence, we assume that there exists a true unobserved spatial process defined at the 12-km grid cell level that is related to both the observational data and the numerical model output via measurement error models. We use the median of the posterior predictive distribution of the unobserved spatial process given the observational data and the CMAQ model output as the daily "fused ambient concentration" of $\mathrm{PM}_{2.5}$. These fused data is defined at the grid cell level as the unobserved spatial process. Since the spatial resolution at which the SHEDS-PM exposure simulator operates is at the census tract level, we have further interpolated our daily fused predictive surfaces of $\mathrm{PM}_{2.5}$ concentration to the centroids of the census tracts considered in this study using ordinary kriging (Cressie, 1993) with an exponential covariance function. The form of the covariance function was determined by a visual inspection of the empirical variogram of the daily fused $\mathrm{PM}_{2.5}$ data, with covariance parameters estimated daily via weighted least squares. We note that the form of the covariance function used to krige the outdoor concentration to the census tract centroids is not expected to influence our findings, since in our model, exposure to $\mathrm{PM}_{2.5}$, only enters the analysis through exposure metrics or through the empirical distributions of personal exposure generated using SHEDS-PM. 
Figure 1(b) displays the average $\mathrm{PM}_{2.5}$ concentration during years 2001-2002 as obtained from the fused surfaces for each census tract considered in this study. As the figure shows, there is considerable spatial variability in the level of air pollution, with the central counties of Wake and Guilford having two of the highest average concentrations. The spatial map also displays some unexpected features, such as the moderate level of $\mathrm{PM}_{2.5}$ in Mecklenburg county, which contains the city of Charlotte, and the elevated concentration of $\mathrm{PM}_{2.5}$ in Vance county. The latter is likely explained by the presence of a power plant nearby.

\section{SHEDS-PM}

The Stochastic Human Exposure and Dose Simulator for PM (SHEDS-PM; Burke et al., 2001) is a stochastic model that estimates the distribution of average daily personal exposure for a population via a two-dimensional Monte Carlo sampling scheme that propagates the uncertainty in the input distributions to the predicted distribution of personal exposure. Several different inputs are needed by the SHEDS-PM model, including probability distributions of exposure factors and three types of databases. The latter include: (1) hourly or daily average of $\mathrm{PM}_{2.5}$ concentration for each census tract in the spatial domain of interest; (2) demographic information for each census tract; and (3) human activity diaries from the publicly available CHAD database with data on the amount of time individuals spend in different locations (i.e., outdoors, indoors, in vehicle). The former include: parameters of equations for estimating indoor PM concentrations, such as residential air exchange rates, house volumes, penetration/deposition rates, and indoor source emission rates (e.g., cooking, cleaning, and smoking). The distributions assigned to each of these inputs have been developed using data from previous studies and may characterize the variability in the data using multiple distributions that depend on season or housing type, for example.

To estimate the distribution of average daily personal exposure for a population, SHEDS-PM simulates for each census tract, a representative number of individuals that match the census tract in demographic characteristics such as gender, age, employment status, and housing type. Each simulated individual is randomly assigned a set of activity diaries for the time period of PM concentration data, based on the individual's personal characteristics, day of the week and season. SHEDS-PM calculates the PM concentration for each location in the individual's activity diary (microenvironment) using the time series of ambient PM concentrations in equations that estimate the contribution due to both outdoor and indoor sources.

More specifically, let $i$ denote a census tract, let $s$ denote an individual simulated by SHEDS-PM in tract $i$, and $C_{i}(d)$ be the average daily $\mathrm{PM}_{2.5}$ concentration in census tract $i$ on day $d$. Then, the ambient exposure or the exposure due to outdoor concentration for individual $s$ living in census tract $i$ on day $d$ is:

$E_{i s}^{a}(d)=C_{i}(d)$

An individual's exposure in each of the seven indoor microenvironments is derived using microenvironment specific equations. In particular, for the residence microenvironment (denoted by $r$ ), the average daily concentration of $P M_{2.5}$ for simulated individual $s$ living in census tract $i$ is obtained using the single-compartment steady-state mass balance equation (Özkaynak et al., 1996):

$$
\begin{aligned}
& C_{i s}^{(r)}(d)=C_{\text {is ambient }}^{(r)}(d)+C_{i s \text { non-ambient }}^{(r)}(d) \\
& =\frac{P_{s} \times a c h_{s}}{a c h_{s}+k_{s}} \cdot C_{i}(d)+\frac{E_{s m k, s} N_{c i g, s}+E_{c o o k, s} t_{c o o k, s}+E_{o t h e r, s}}{\left(a c h_{s}+k_{s}\right) V_{i s} T}
\end{aligned}
$$

where $P_{s}, k_{s}, a c h_{s}$, and $V_{i s}$ denote, respectively, the penetration factor, the deposition rate, the air exchange rate, and the volume of the residence of simulated individual $s$ living in census tract $i$, while $E_{s m k, s}, N_{c i g, s}, E_{c o o k, s}, t_{c o o k, s}$, and $E_{\text {other,s }}$ indicate, respectively, the emission rate for smoking, the numbers of cigarette individual $s$ smoked during the SHEDS-PM model time step $T$, the emission rate for cooking, the time simulated individual $s$ spent cooking during model time step $T$, and the emission rate for other sources for simulated individual $s$.

On the other hand, the average daily concentration of $P M_{2.5}$ in any other indoor microenvironment $m$ different from residence (e.g., office, school, store, restaurant, bar, and vehicle) is given by:

$C_{i s}^{(m)}(d)=b_{0 s}^{(m)}+b_{1 s}^{(m)} C_{i}(d)$

where $b_{0 s}^{(m)}$ and $b_{1 s}^{(m)}$ represent, respectively, the contributions to average concentration in microenvironment $m$ due to indoor and outdoor sources. The regression coefficients in Equation (3) are provided with probability distributions derived from previous exposure studies, and are randomly sampled for each simulated individual. Note that, while in theory the average daily concentration of $\mathrm{PM}_{2.5}$ in any indoor microenvironment could be computed using a similar mass balance equation to Equation (2), in practice, the scarcity of data available for other microenvironments renders estimating the mass-balance equation parameters (i.e., air exchange rate, penetration, deposition rate, etc.) infeasible for the non-residential microenvironments, hence motivating the regression in Equation (3).

The average daily personal exposure for a simulated individual $s$ is computed by summing the individual's exposure to ambient concentration and the individual's exposure in each of the seven indoor microenvironments. At their turn, each of these terms is obtained 
by multiplying, respectively, the average daily concentration of particulate matter either outdoor or in any of the seven indoor microenvironments by the amount of time the individual spends outdoor or in any of the indoor microenvironments.

More specifically, revising (1), if $m=1, \ldots, 7$ denotes an indoor microenvironment and $C_{i s}^{(m)}(d)$ indicates the average daily concentration of particulate matter in indoor microenvironment $m$ for individual $s$, with $C_{i s}^{(1)}(d)=C_{i s}^{(r)}(d)$, the average daily concentration of $\mathrm{PM}_{2.5}$ in the residential microenvironment, then the average daily personal exposure for individual $s$ is given by:

$E_{i s}^{p}(d)=w_{i s}^{(o)}(d) \cdot C_{i}(d)+\sum_{m=1}^{7} w_{i s}^{(m)}(d) \cdot C_{i s}^{(m)}(d)$

with $w_{i s}^{(o)}(d)$ the fraction of time individual $s$ spends outdoors and $w_{i s}^{(m)}$ the fraction of time individual $s$ spends in indoor microenvironment $m, m=1, \ldots, 7$.

Finally, the distribution of average daily personal exposure on day $d$ for a population is obtained as the empirical distribution of average daily personal exposure for each simulated individual. In addition to the average daily personal exposure for each simulated individual, SHEDS-PM also returns the decomposition of the exposure in terms of outdoor and indoor sources.

\section{CHARACTERIZING EXPOSURE}

During the course of a pregnancy, a woman undergoes physiological, biochemical and anatomical changes that might affect her overall response and susceptibility to external stimuli. To investigate whether there are periods during the course of a pregnancy in which a mother and her fetus are more vulnerable to the harmful effects of $\mathrm{PM}_{2.5}$, and to determine which aspects of the exposure to $\mathrm{PM}_{2.5}$ are more critical, we characterize maternal exposure to $\mathrm{PM}_{2.5}$ using different metrics examined in different time windows.

We can develop such metrics because of the high spatio-temporal resolution of our air pollution data. Normally, in studies of air pollution and birth outcomes, researchers have used only monitoring data to represent exposure. Due to the infrequent and irregular sampling of $\mathrm{PM}_{2.5}$ monitors and their sparse locations, most researchers have used as exposure metric the ambient average concentration reported by monitors located within a county (Chen et al., 2002; Basu et al., 2004; Bell et al., 2007), an area (Lee et al., 2003; Liu et al., 2003,Wang et al., 1997; Woodruff et al., 1997), or a city (Ha et al., 2001; Maisonet et al., 2001; Gouveia et al., 2004; Mannes et al., 2005; Hansen et al., 2006). Others have used as exposure metric the average obtained from the closest monitor(s) to the subject's residence, but limiting their analysis only to birth outcomes for mothers who reside within a certain distance from monitoring sites (Ritz and Yu, 1999; Ritz et al., 2000; Yang et al., 2003; Basu et al., 2004; Lin et al., 2004; Mannes et al., 2005; Parker et al., 2005; Dugandzic et al., 2006). In our case, the high spatio-temporal resolution of the air pollution data, permits us to use all the birth outcome data and allows us to investigate several research questions. More specifically, we can investigate whether it is the prolonged maternal exposure to $\mathrm{PM}_{2.5}$ or the exposure to extreme values of $\mathrm{PM}_{2.5}$ or the combined effect of both that is more harmful to the fetus.

To describe the three different metrics that we have developed, we follow the notation introduced in Section 3 , denoting with $C_{i}(d)$ the average daily $\mathrm{PM}_{2.5}$ concentration in census tract $i$ on day $d$. We indicate with $T_{i j}$ the period of long-term exposure for individual $j$ living in census tract $i$, while we use the symbol $W_{i j}$ to indicate any sub-window of exposure, i.e., $W_{i j} \subseteq T_{i j}$. In the context of our study, the period of long-term exposure $T_{i j}$ coincides with subject $j$ 's entire pregnancy; in other environmental exposure studies, the definition of the period of long-term exposure will be different and might not be subject-specific. As in Equation (1), the daily ambient exposure for individual $j$ on day $d$ is given by $E_{i j}^{a}(d)=C_{i}(d)$. This implies that the ambient long-term exposure for individual $j$ during $T_{i j}$ is given by the time series:

$E_{i j}^{a}=\left\{E_{i j}^{a}(d): d \in T_{i j}\right\}=\left\{C_{i}(d): d \in T_{i j}\right\}$

With regard to individual exposure, we used SHEDS-PM to associate with each pregnancy $j$ in tract $i$ an empirical distribution of daily personal exposure, $E_{i j}^{p}(d)$. More precisely, if we denote with $S_{i j}=\left\{s_{j}\right\}$ the set of individuals $s_{j}$ simulated by SHEDS-PM in census tract $i$ that match subject $j$ in terms of age, then we obtain the set of daily personal exposures $\left\{E_{i s_{j}}^{p}(d): s_{j} \in S_{i j}\right\}$ derived according to Equation (4). In turn, we obtain the personal long-term exposure for individual $j$ during $T_{i j}$ as a set of time series, $\left\{E_{i s_{j}}^{p}: s_{j} \in S_{i j}\right\}$ where

$E_{i s_{j}}^{p}=\left\{E_{i s_{j}}^{p}(d): d \in T_{i j}\right\}$

In our study, for each $i$ and $j$ we took the set $S_{i j}$ to be of cardinality $n=30$, while we set the average daily ambient concentration $C_{i}(d)$ of $\mathrm{PM}_{2.5}$ in census tract $i$ on day $d$ to be equal to the fused daily concentration of $\mathrm{PM}_{2.5}$. The proposed metrics below compute a function of $E_{i j}^{a}$ to obtain the ambient value of the metric and also compute this function for each of the $E_{i s_{j}}^{p}$ to obtain an empirical distribution of personal exposure under this metric.

Figure 2(a) shows the time series $E_{i j}^{a}$, the ambient exposure curve for an illustrative mother in our database living in Mecklenburg county whose pregnancy $T_{i j}$ spans the period from January 20 to October 27, 2001. On the other hand, Figure 2(b) displays, for each day $d$ in $T_{i j}$ the range of the set $\left\{E_{i s_{j}}^{p}(d): s_{j} \in S_{i j}\right\}$ for the same mother considered in Figure 2(a). The set of $E_{i s_{j}}^{p}$, s, the set of personal exposure curves for individual $j$ are shown in Figure 2(b).

The vertical lines in Figure 2(a) and Figure 2(b) indicate seven illustrative time windows $W_{i j}$ that we have considered in our analysis for each $i, j$ : respectively, the first 30 days, the first 60 days, the first trimester, the second trimester, the last 60 days, the last 30 days, and the entire pregnancy, $T_{i j}$. Each of the metrics that we present below have been examined through these time windows. 

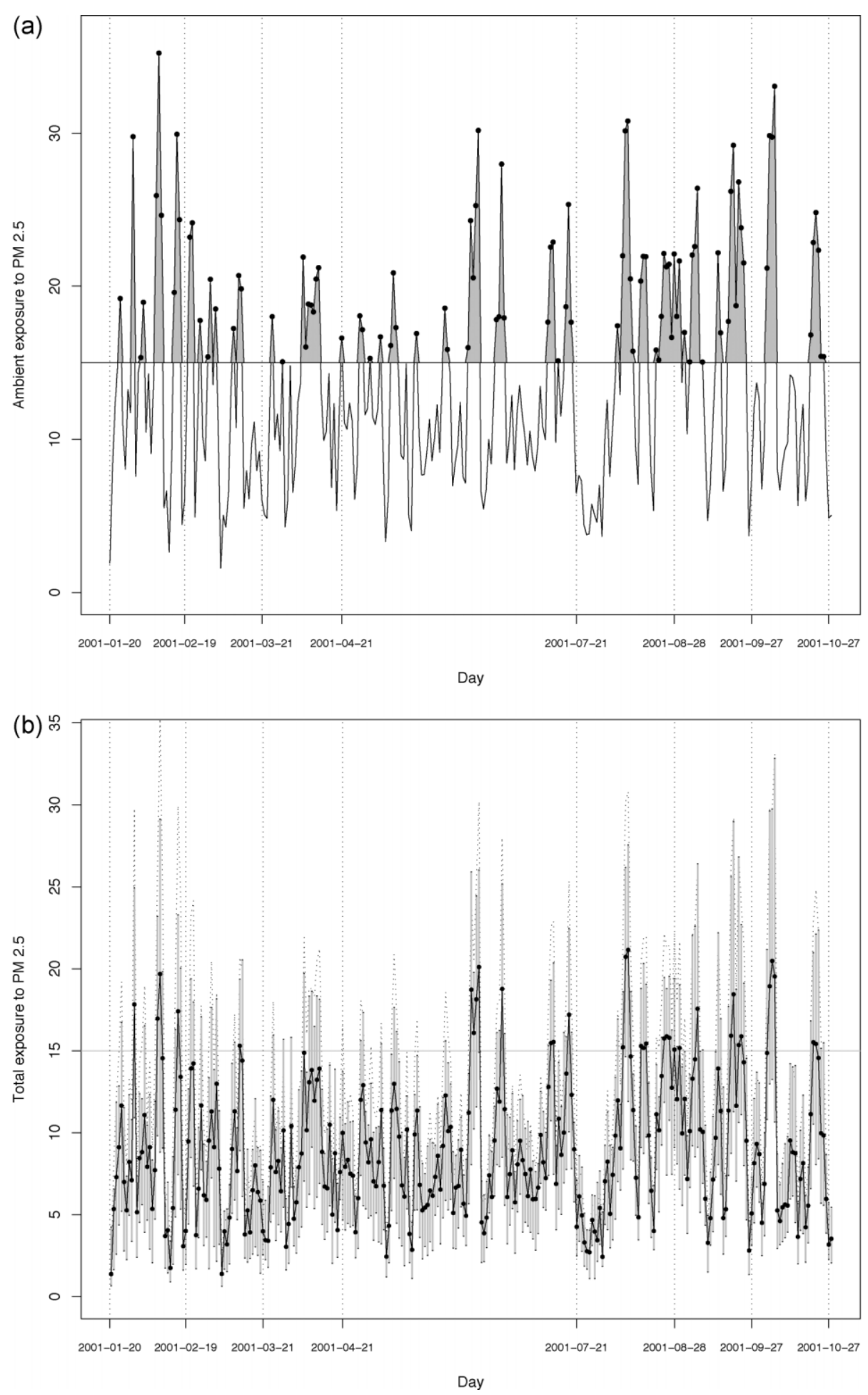

Figure 2. (a) Ambient exposure curve or daily exposure to "fused" ambient $\mathrm{PM}_{2.5}$ concentration during the entire course of the pregnancy for a woman living in a census tract in Mecklenburg. (b) Range of the 30 daily personal exposure values associated to the woman in consideration obtained from the SHEDS-PM simulator (gray vertical parallel lines) and median of the 30 daily personal exposure values (black solid line and black dots)

\subsection{First metric: average exposure}

Following previous studies on air pollution and birthweight, we take as a first metric the average exposure to $\mathrm{PM}_{2.5}$ over any time window $W_{i j}$. Thus, under the ambient exposure scenario, the first metric, the average ambient exposure, is given by

$\mathcal{A}_{W_{i j}}^{a}=\frac{1}{\left|W_{i j}\right|} \sum_{d \in W_{i j}} E_{i j}^{a}(d)$ 
where $\left|W_{i j}\right|$ denotes the length of the time window $W_{i j}$. For the illustrative mother in Mecklenburg, the value of this metric over the entire pregnancy is $13.36 \mu \mathrm{g} / \mathrm{m}^{3}$. Under the personal exposure scenario the average personal exposure, $\mathcal{A}_{W_{i j}}^{p}$, is estimated using the set $\left\{\mathcal{A}_{W_{i s_{j}}}^{p}: s_{j} \in \mathcal{S}_{i j}\right\}$, where, for each simulated individual $s_{j} \in \mathcal{S}_{i j}, \mathcal{A}_{W_{i s_{j}}}^{p}$ is computed using the analogous version of Equation $(5)$ with $E_{i j}^{a}(d)$ being replaced by $E_{i s_{j}}^{p}(d)$. Thus, under the personal exposure scenario, the first metric is estimated with a distribution. Figure 3(a) displays a kernel density estimate of this distribution when the time window of exposure is the entire pregnancy. The value of the average ambient exposure $\mathcal{A}_{T_{i j}}^{a}$ during the entire pregnancy $T_{i j}$ is indicated in Figure 3(a) with a vertical line.

\subsection{Second metric: percentage of days over a threshold}

The second metric finds its justification in the hypothesis that perhaps exposure to high values of $\mathrm{PM}_{2.5}$ is particularly deleterious for birthweight; average exposure does not capture this. Therefore, for individual $j$ living in census tract $i$, we define percentage of days daily ambient exposure exceeded a threshold level $\lambda$ during time window $W_{i j}$, the quantity,

$P_{W_{i j}, \lambda}^{a}=\left(\frac{1}{\left|W_{i j}\right|} \sum_{d \in W_{i j}} 1_{\left(E_{i j}^{a}(d) \geq \lambda\right)}\right) \times 100$

where $\mathbf{1}_{\left(E_{i j}^{a}(d) \geq \lambda\right)}$ is the indicator function for $E_{i j}^{a}(d) \geq \lambda$ or not. Note that in Equation (6), we standardize the metric to account for differences in the length of pregnancies.

Given the frequency distribution of daily $\mathrm{PM}_{2.5}$ concentration in the census tracts in North Carolina considered in this study, we took as threshold $\lambda$ the National Ambient Air Quality Standard (NAAQS) set by EPA for particulate matter for the yearly average, that is, $15 \mu g / \mathrm{m}^{3}$. Using this threshold, the value of the second metric for the illustrative mother in Mecklenburg is equal to $35.92 \%$ when $W_{i j}=T_{i j}$. In an analogous way, we define the second metric under the personal exposure scenario: the percentage of days daily personal exposure exceeded a threshold $\lambda, P_{W_{i j}, \lambda}^{p}$. As for the first exposure metric, we estimate $P_{W_{i j}, \lambda}^{p}$ using the set $\left\{P_{W_{i s_{j}}, \lambda}^{p}: s_{j} \in \mathcal{S}_{i j}\right\}$ where $P_{W_{i s_{j}}, \lambda}^{p}$ has been computed, for
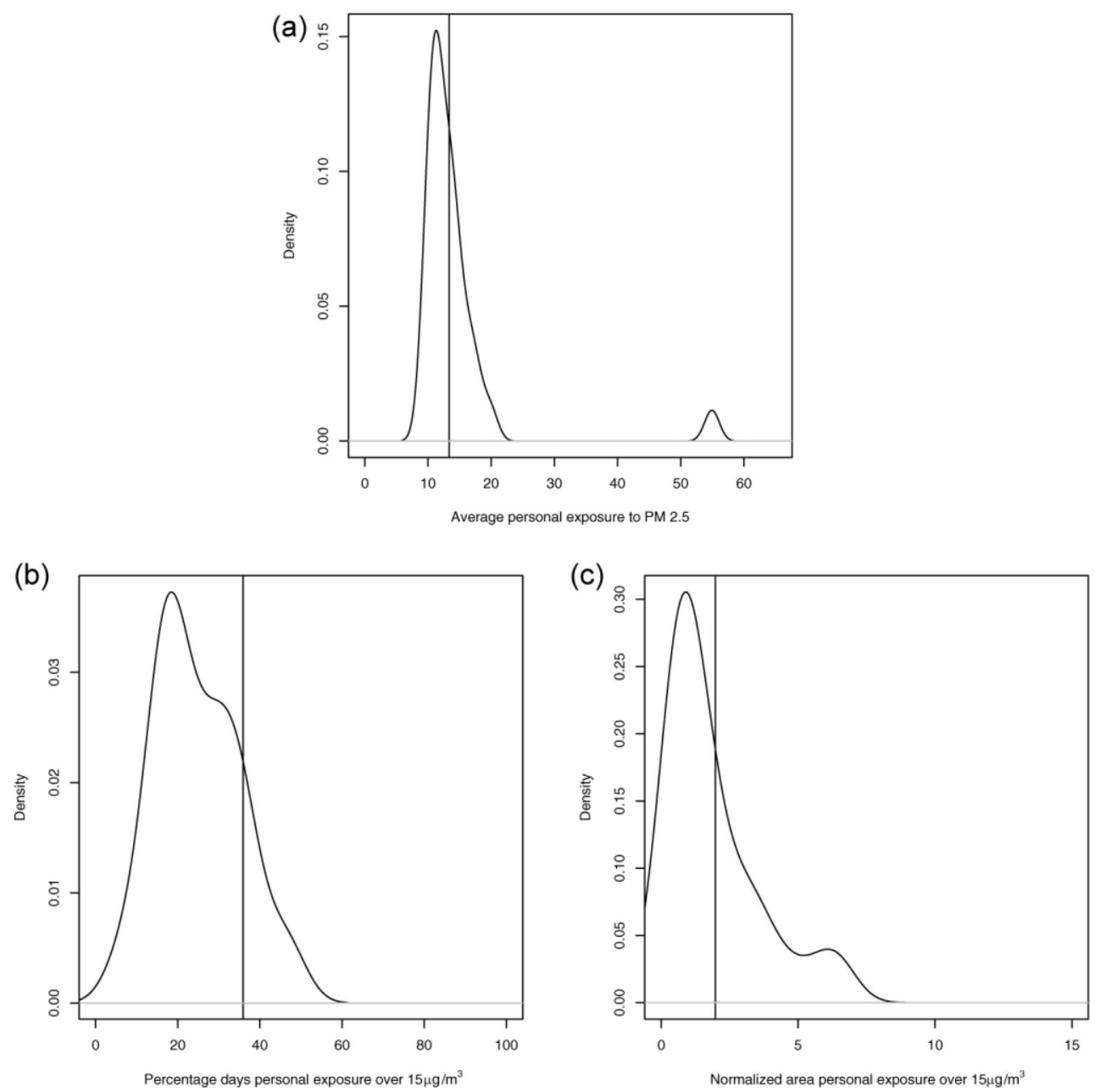

Figure 3. (a) Distributions of average personal exposure to $\mathrm{PM}_{2.5}$ during the entire pregnancy for the mother considered in Figure 2(a). (b) Distribution of the percentage of days personal exposure to $\mathrm{PM}_{2.5}$ exceeded $15 \mu \mathrm{g} / \mathrm{m}^{3}$ during the entire pregnancy for the mother considered in Figure 2(a). (c) Distributions of the normalized area under the personal exposure curve above $15 \mu \mathrm{g} / \mathrm{m}^{3}$ during the entire pregnancy for the mother considered in Figure 2(a). In panels (a)-(c) the vertical line display the value of the corresponding three metrics obtained using ambient exposure 
each $s_{j} \in \mathcal{S}_{i j}$ by using an appropriately modified version of Equation (6). A kernel density estimate of the distribution defined by the set $\left\{P_{W_{i s}, \lambda}^{p}: s_{j} \in S_{i j}\right\}$ for the illustrative mother living in Mecklenburg is presented in Figure 3(b), with the vertical line displaying the value of the second metric under the ambient exposure approach. Here, the time window of exposure considered is the entire pregnancy $T_{i j}$.

\subsection{Third metric: area above a threshold}

The second metric, while accounting for extremes, does not take into consideration their magnitude. Additionally, it does not differentiate between situations where high concentration of particulate matter is an isolated event and situations where the elevated exposure is prolonged over time. To take into account both of these aspects, magnitude and length of duration, we define a third metric, the area under the subject's exposure curve above a threshold $\lambda$ during a selected time window $W_{i j}$. Also in this case, to account for differences in gestation lengths, we have standardized the metric by dividing it by the length of the time window. Therefore, in the case of ambient exposure, for any window of exposure $W_{i j}$, we define the normalized area under the ambient exposure curve above $\lambda$ as the quantity,

$\mathcal{R}_{W_{i j}, \lambda}^{a}=\frac{1}{\left|W_{i j}\right|} \sum_{d \in W_{i j}}\left(1_{\left(E_{i j}^{a}(d) \geq \lambda\right)}\right) \cdot\left(E_{i j}^{a}(d)-\lambda\right)$.

Analogously, we define the normalized area under the personal exposure curve above $\lambda, \mathcal{R}_{W_{i j}, \lambda}^{p}$, which we estimate via the distribution defined by the set $\left\{\mathcal{R}_{W_{i s_{j}}, \lambda}^{p}: s_{j} \in \mathcal{S}_{i j}\right\}$. For the illustrative mother in consideration, taking $\lambda=15 \mu \mathrm{g} / \mathrm{m}^{3}$, the normalized area under the ambient exposure curve above $\lambda$ when $W_{i j}=T_{i j}$ is $1.98\left(\mu \mathrm{g} / \mathrm{m}^{3}\right)^{2}$, while the kernel density estimate of the distribution defined by the set $\left\{\mathcal{R}_{W_{i s}, \lambda}^{p}: s_{j} \in \mathcal{S}_{i j}\right\}$ is displayed in Figure $3(\mathrm{c})$.

Thus, under the ambient exposure scenario, to each mother is associated a set of $3 \times 7=21$ values, given by the three metrics evaluated over the seven time windows. Correspondingly, using personal exposure, to each mother is associated a set of 21 distributions.

Figure 3 presents kernel density estimates of three of these distributions for the illustrative mother that we took as example. In each panel, the corresponding value of the metric obtained using ambient exposure is indicated by a vertical line. As these plots seem to indicate, for this particular individual, the value of the exposure metrics obtained using ambient exposure are either about the same magnitude or larger than the median of the distributions of the corresponding exposure metrics obtained using the personal exposure curves. This is true for most of the subjects in our study as pairwise scatterplots of the value of the three exposure metrics obtained using ambient exposure and of the median of the distributions of the corresponding exposure metrics obtained using the personal exposure curves when the time window of exposure is the entire pregnancy indicate (figure not shown).

To further illustrate the relationship between the values of the exposure metrics obtained using ambient exposure and the distributions of those metrics obtained under the personal exposure scenario, Figure 7 in the Appendix shows kernel density estimates of the distributions of the three metrics for all the women in our database grouped by age when the selected time window of exposure is the entire pregnancy. More specifically, Figure 7(a) presents the distribution of the average ambient exposure metric, while panel (c) shows the distribution of the average personal exposure metric. To construct the distribution presented in panel (b) we have considered, for each woman, the median of the distribution of the average personal exposure metric. We have proceeded similarly for the other two metrics.

In accord with what was observed in Figure 3, the average ambient exposure metric and the median of the distribution of average personal exposure are fairly comparable in terms of magnitude and spread. On the other hand, the distribution of average personal exposure is more spread out than that of average ambient exposure as panel (c) in Figure 7 indicate, allowing for the possibility of high personal exposures, which is not contemplated in the ambient exposure scenario. Also, as a natural consequence, the range of possible values for the second and third metrics are larger under the personal exposure approach than in the ambient exposure scenario, as panels (e) and (h) of Figure 7 show. Additionally, while ambient exposure doesn't present any difference in the profile of the distributions for the different age groups, the distributions of the three metrics are different for the four age groups when exposure is characterized in terms of personal exposure. In particular, from Figure 7 it appears that, in general, personal exposures of younger mothers are lower than that of older mothers.

\section{THE MODEL}

We start by presenting the model employed in the ambient exposure scenario and we subsequently show how the model is modified to incorporate a distribution for personal exposure. In both cases, the spatial unit at which we work is the census tract, a total of 616 in our study region.

\subsection{Using ambient exposure}

Let $i$ be the index denoting a census tract in our area of study, with $i=1, \ldots, M=616$, and let $Y_{i j}$ denote the birthweight of a baby born to mother $j$ living in census tract $i, j$ ranging from 0 to $N_{i}, N_{i}$ being the number of subjects in our birth outcome dataset living in census tract $i$. Let $X_{i j}$ be a vector with socio-economic information for subject $j$ living in census tract $i$. $X_{i j}$ contains information on: gestation length, measured in weeks, maternal race, categorized into three classes (Non-Hispanic White, Non-Hispanic Black, and Hispanics), maternal age, grouped into four classes (15-17, 18-24, 25-34, and 35-44 years of age), maternal education, categorized into five classes (middle school, some high school, high school, some college, and college), maternal smoking status, grouped into two classes (smoker and non-smoker), 
marital status (married and not-married), birth order, grouped into two categories (first born and not-first born), and sex of the baby. Let $O_{i}$ denote the percentage of owner-occupied units in census tract $i$ (other tract level covariates were considered but were not significant). In performing our analysis we take as baseline the following classes: Non-Hispanic White for maternal race, 25-34 for maternal age class, high school for maternal eduction, non-smoking for smoking status, married for marital status, non-first born for birth order, and female for sex of the baby. Differently from some studies on the relationship between maternal exposure to a pollutant and birthweight (Woodruff et al., 1997; Ritz and Yu 1999; Ritz et al., 2000; Chen et al., 2002; Yang et al., 2003; Basu et al., 2004; Parker et al., 2005; Hansen et al., 2006), our model includes also gestation length. As Dugandzic et al. (2006); Bell et al. (2007); Wang et al. (1997); Maisonet et al. (2001); Liu et al. (2003); Lin et al. (2004); Lee et al. (2003); Gouveia et al. (2004); Ha et al. (2001); Mannes et al. (2005); Bobak (2000), we adjust for gestation length because the length of the gestation period is, evidently, a good predictor of birthweight. Finally, we let $z_{i j}$ denote one of the exposure metrics defined in Section 4 for mother $j$ living in census tract $i$ evaluated over one of the seven time windows $W_{i j}$. Thus, $z_{i j}$ is either $\mathcal{A}_{W_{i j}}^{a}, P_{W_{i j}, \lambda}^{a}$, or $\mathcal{R}_{W_{i j}, \lambda}^{a}$.

Then, our model relates ambient exposure to $\mathrm{PM}_{2.5}$ and birthweight in the following way:

$Y_{i j}=\beta_{0}+X_{i j} \beta+\alpha O_{i}+\gamma z_{i j}+\phi_{i}+\varepsilon_{i j}$

for $i=1, \ldots, M$ and $j=1, \ldots, N_{i}$ with $\beta_{0}$ representing an intercept term, $\beta$ a vector of coefficients for the socio-economic factors, $\alpha$ a coefficient of the effect of $O_{i}$, the percentage of owner-occupied units in census tract $i, \phi_{i}$ a spatial random effect for census tract $i$ and $\varepsilon_{i j}$ an error term that follows a normal distribution with mean zero and variance $\sigma^{2}$. We include $\phi_{i}$ in Equation (8) to model spatial variability between census tracts. Even though the ambient exposure term might account for some of the spatial variability in the response variable, we introduce spatial random effects in the model because we anticipate that there is additional residual spatial variability in the data at the tract level that is not accounted for by $O_{i}$. We note that we also fitted all of the models in the sequel without random effects, essentially using ordinary least squares (OLS). Summarizing, in the interest of space, these models do not predict as well (Table 2 below); introducing a spatially-varying intercept for the census tracts provides improved model flexibility over a constant intercept. Figure 4 below reveals the substantial spatial variability in these intercepts and their consequential magnitude in grams of birthweight. Expressed in different terms, location matters though we do not know the spatial variables that account for this.

Evidently, an enormous number of models can be considered by varying exposure metric, choice of window associated with the metric, and including one or more metrics. Here we present results for an illustrative set of choices. Precisely, if the time window of exposure $W_{i j}$ is the entire pregnancy $T_{i j}$, then $z_{i j}$ can be, respectively, the average ambient exposure during the entire pregnancy, $\mathcal{A}_{T_{i j}}^{a}$, the percentage of days during the entire pregnancy ambient exposure exceeded $15 \mu \mathrm{g} / \mathrm{m}^{3}, P_{T_{i,}, 15}^{a}$, or the normalized area under the ambient exposure curve above $15 \mu \mathrm{g} / \mathrm{m}^{3}, \mathcal{R}_{T_{i j}, 15}^{a}$. However, if the time window is any of the subsets $W_{i j} \subset T_{i j}$, then the exposure metrics appear in the model equation as a pair. More specifically, given an exposure metric we will introduce simultaneously in the model the value of the exposure metric evaluated over the first 30 and last 30 days of the pregnancy, for example, or over the first and last 60 days of the pregnancy, or over the first and second trimester. Hence, Equation (8) presents the model for birthweight and ambient exposure when the time window of exposure $W_{i j}=T_{i j}$. For other time windows $W_{i j} \subset T_{i j}$, Equation (8) should be modified as:

$Y_{i j}=\beta_{0}+X_{i j} \beta+\alpha O_{i}+\gamma_{1} z_{i j 1}+\gamma_{2} z_{i j 2}+\phi_{i}+\varepsilon_{i j}$

where $z_{i j 1}$ and $z_{i j 2}$ indicate the two exposure metrics for mother $j$ living in census tract $i$ evaluated over the two "paired" time windows. In total, we consider 12 models, four for each exposure metric.

We complete the specification of model (8) (respectively, (9)) by assigning prior distributions to the parameters in the model. Precisely,

$$
\begin{gathered}
\beta_{0} \sim N\left(\mu_{0}, v_{0}^{2}\right) \\
\beta \sim \operatorname{MVN}(\mu, V) \\
\alpha \sim N\left(\mu_{\alpha}, v_{\alpha}^{2}\right) \\
\gamma \sim N\left(\theta, \tau^{2}\right) \\
\sigma^{2} \sim \operatorname{InvGamma}(\eta, v)
\end{gathered}
$$

while the spatial random effects for the census tract are modeled using a CAR model (Besag 1974; Banerjee et al., 2004). The hyperparameters for the residual variance $\sigma^{2}$ were determined as follows: the shape parameter $\eta$ was set equal to 2.1, so that the variance of the Inverse Gamma exists but it is rather large, while the scale parameter $v$ was set equal to the estimate of the residual variance obtained when fitting a linear regression model to birthweight using data from previous years and including in the model the same individual-level and tract-level covariates considered in our analysis, but excluding the exposure variables and the spatial random effects.

The prior specification for model (9) involves replacing the univariate normal distribution for $\gamma$ with a bivariate normal distribution for $\gamma_{1}$ and $\gamma_{2}$. Hyperparameters of the priors were chosen to have proper but diffuse priors. Estimates of the parameters in the model are obtained via MCMC using a Gibbs sampling algorithm (Gelfand and Smith 1990), with "centering on the fly" after each model-fitting iteration (Besag et al., 1995; Gelfand et al., 1996) to convert the improper CAR specification to a proper one.

The code to implement the MCMC algorithm has been written in $\mathrm{C}++$ and it runs in 8 to $10 \mathrm{~h}$ depending on whether the exposure metrics enter the model as a single value/distribution or as a pair of values/distributions.

\subsection{Using personal exposure}

Using personal exposure, we have a set of 21 distributions for the combinations exposure metric-time window of exposure. Therefore, in the personal exposure framework, models (8) and (9) need to be appropriately modified to accommodate for these distributions. We achieve this by modifying the second stage specification of models (9) and (10). More specifically, while Equations (8) and (9) still hold, we add to 

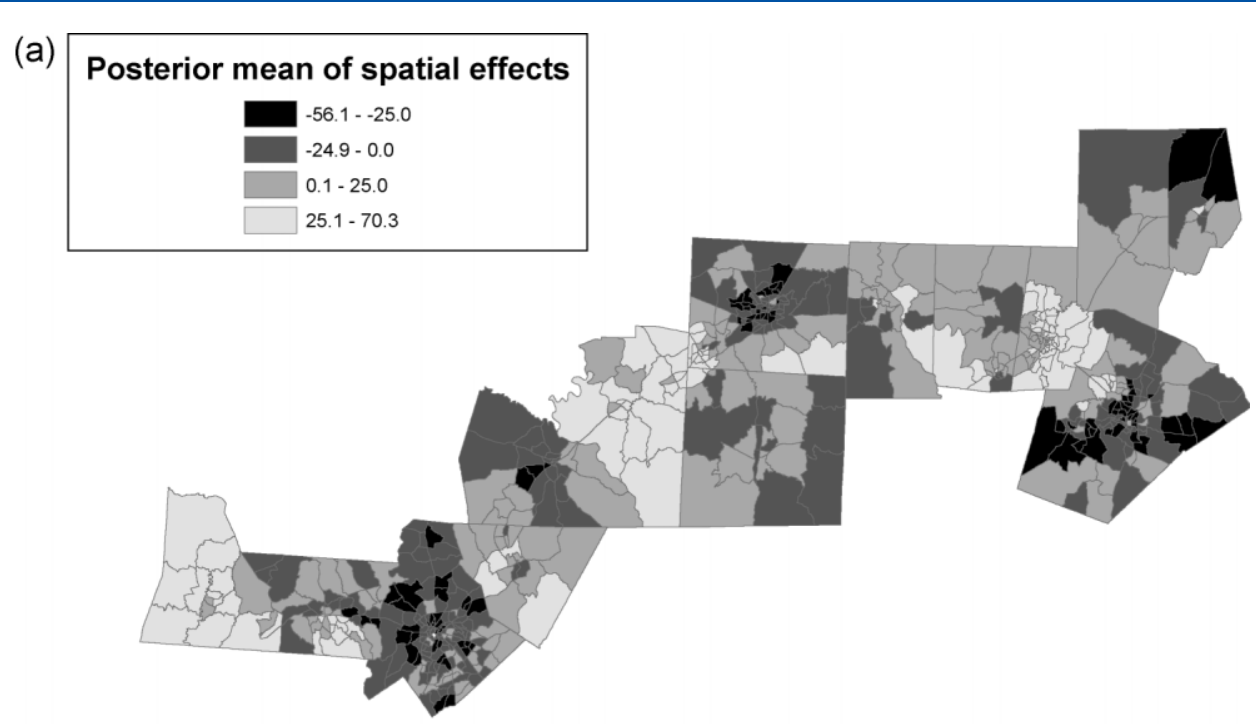

(b)

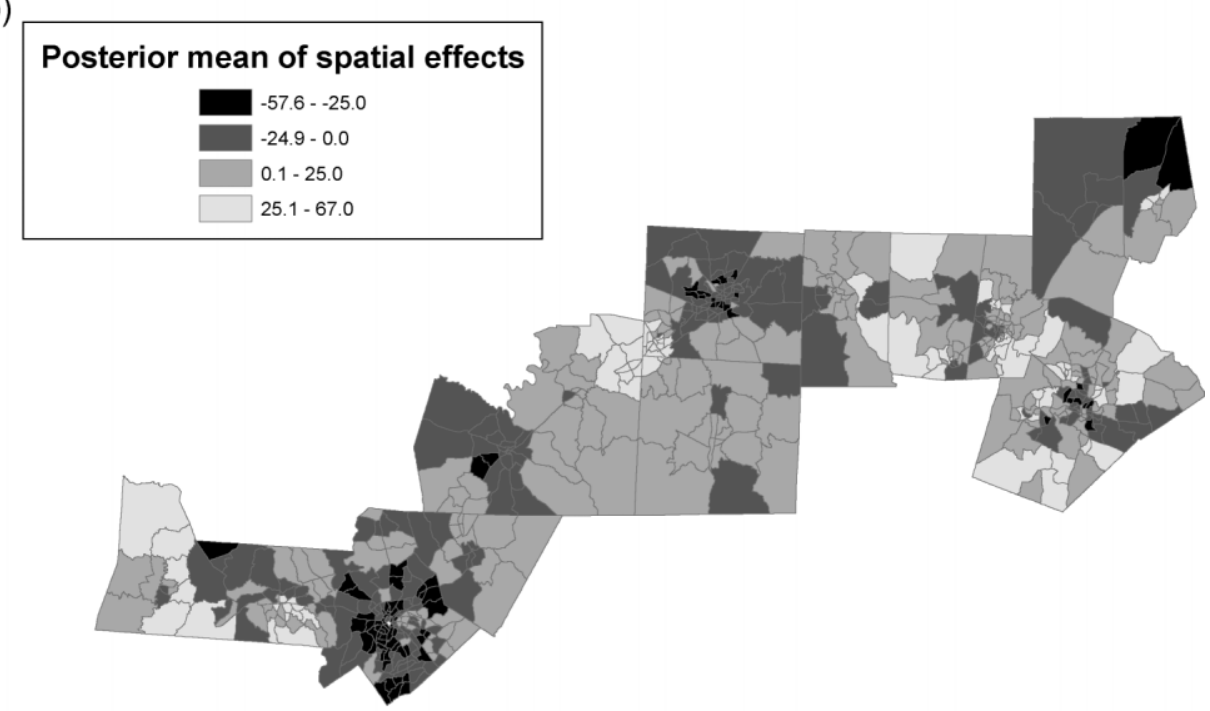

Figure 4. Posterior means of the random spatial effects obtained from the model where the exposure metric is: (a) "normalized area under the ambient exposure curve above $15 \mu \mathrm{g} / \mathrm{m}^{3}$ " and (b) "normalized area under the personal exposure curve above $15 \mu \mathrm{g} / \mathrm{m}^{3}$ ". In both models, the time window of exposure is the entire pregnancy. Additionally, both posterior means are reported in grams

Equation (10) this additional distribution:

$z_{i j} \sim \operatorname{Uniform}_{30}\left(Z_{i j}\right)$

where Uniform 30 denotes the discrete uniform distribution on a set of 30 values with support $Z_{i j}$ which changes for $i=1, \ldots, M$ and $j=1, \ldots, N_{i}$, and is given respectively by $\left\{A_{W_{i s_{j}}}^{p}: s_{j} \in S_{i j}\right\},\left\{P_{W_{i s}}^{p}: s_{j} \in S_{i j}\right\}$, or $\left\{R_{W_{i s_{j}}}^{p}: s_{j} \in S_{i j}\right\}$ for any $W_{i j} \subseteq T_{i j}$.

As under the ambient exposure scenario, exposure metrics enter the model as a pair if the time windows considered are the first and last 30 days of the pregnancy, the first and last 60 days of the pregnancy, and the first and second trimester. In these cases, we add two additional distributions $z_{i j 1} \sim \operatorname{Uniform}_{30}\left(Z_{i j 1}\right)$ and $z_{i j 2} \sim \operatorname{Uniform}_{30}\left(Z_{i j 2}\right)$ to the specification of the second stage (10). Again, the resulting 12 models were fit via MCMC using a Gibbs sampling algorithm.

\section{RESULTS}

Across all models, the estimates of the coefficients of the socio-economic factors are rather consistent and consistent with previous findings. Additionally, with the exception of percentage of owner-occupied units in the census tract of maternal residence, marital status, and maternal age between 35 and 44, all of the coefficients are significant for all the models. The significance of the coefficient for the percentage of owner-occupied units in the census tract of maternal residence and marital status varies from model to model.

As previously commented, gestation length is probably the best predictor of birthweight and all models seem to indicate that for each additional week of gestation, birthweight increases of about 180-185 grams. All of our models indicate that Non-Hispanic Black women 
deliver babies that are on average about 165-180 grams lighter than babies of Non-Hispanic White mothers, while babies of Hispanic women are only 60-65 grams lighter than babies of Non-Hispanic White mothers. Lower levels of maternal education are negatively associated with birthweight: mothers with middle school education have babies that are on average 40-50 grams lighter than those of mothers with a high school education, while mothers that did not complete high school deliver babies that are on average 35-40 grams lighter than those of mothers who completed it. The situation is reversed for mothers with higher levels of education: birthweight increases by about 24-30 grams if the mother has done some college and by 25-35 grams if the mother has a college degree. Being too young at delivery also appears to be detrimental to birthweight. In particular, compared to the birthweight of a baby from a mother who is $25-34$ years old at delivery, birthweight decreases by about 35-50 grams if the mother is between 15 and 17 years of age at delivery, and by 30-45 grams if maternal age is between 18 and 24 years of age. No significant difference in birthweight is found between mothers who deliver at 25-34 years of age and mothers who deliver at 35-44 years of age. Smoking and not being married are also risk factors. In particular, smoking during pregnancy is associated with a decrease of 180-190 grams, while not being married, in some models, is associated with a decrease of 30-40 grams in birthweight. Finally, not being the first baby and being a male are both associated with a significant increase in birthweight of about 120-135 grams.

Estimates of the coefficients of maternal exposure to particulate matter for some illustrative exposure windows are reported in Table 1. For each combination of exposure metric and time window of exposure, the table presents, in grams, posterior means and the $95 \%$ credible interval obtained by running the models under the ambient exposure scenario and under the personal exposure framework. As Table 1 shows, none of the coefficients of exposure is significant. There are several possible interpretations for such result. More likely, as noted in Section 2.2, it is due to the moderate exposure levels in North Carolina compared with other regions used in such studies. Additionally, it is possible that the relationship between maternal exposure and birthweight is more complicated than just a simple linear relationship as it is implied in our model, or it is possible that the spatial random effects for the census tract of maternal residence are accounting for part of the effect of maternal exposure. It is also important to note the difference in the sign of the coefficient for maternal exposure between models that use ambient exposure to characterize an individual's exposure to $\mathrm{PM}_{2.5}$ and models that use personal exposure. With the exception of the model that introduces maternal exposure via the second metric (percentage of days maternal exposure, whether ambient or personal, exceeded $15 \mu \mathrm{g} / \mathrm{m}^{3}$ ), every time the model using ambient exposure reports a negative (yet not significant) coefficient, the corresponding model using personal exposure reports a positive, yet not significant, coefficient. We believe that this is explained mostly by the different estimates of the spatial random effects obtained under the two models.

Figure 4 and Figure 8, in the Appendix, show, respectively, the posterior means and standard deviations for the spatial random effects obtained from the model that employs the third metric, that is, the normalized area under the ambient exposure curve, respectively, the personal exposure curve, above $15 \mu \mathrm{g} / \mathrm{m}^{3}$ with $W_{i j}=T_{i j}$. We present results for this model because we believe that this metric has the advantage of combining both aspects of the first and second metrics, as it accounts for both magnitude and exceedances.

In Figure 4 darker shades are used to represent negative values, while the two lighter tones are used to represent positive values. As the figure shows, the two models imply a different spatial structure for the spatial random effects. In particular, the model employing ambient exposure seems to indicate more extreme values, both positive and negative, for the spatial random effects, while the spatial map for the model that employes personal exposure indicates more moderate values. Additionally, while both models associate large negative effects to census tracts in Mecklenburg, the two models disagree on the effects associated with census tracts in Wake county. In particular, while the model using personal exposure estimates the effect of some of the tracts in Wake county to be positive and between 0 and 25 grams, according to the model for ambient exposure, babies born from mothers residing in those census tracts are on average 25 to 56 grams lighter. Similar discrepancies can be observed in some of the western tracts in Randolph county: while the model

Table 1. Posterior means and $95 \%$ credible intervals for the coefficients of the different exposure metrics obtained using the two different approaches: the first one characterizes exposure using ambient exposure and the second one characterizes exposure using personal exposure. Covariates included in the model: gestation, maternal age, maternal race, maternal education, marital status, maternal smoking status, sex, birth order, percentage of owner-occupied units in the census tract, and spatial random effect for the census tract of maternal residence. Each coefficient is reported in grams

\begin{tabular}{|llrr|}
\hline Exposure metric & Time window & Ambient exposure & Personal exposure \\
\hline Average exposure & First 30 days & $-0.52(-1.93 ; 0.86)$ & $1.09(-0.13 ; 2.35)$ \\
& Last 30 days & $-0.51(-1.92 ; 0.88)$ & $1.10(-0.12 ; 2.36)$ \\
& First 60 days & $-0.64(-2.21 ; 0.91)$ & $1.23(-0.22 ; 2.59)$ \\
Percentage days daily exposure over $15 \mu \mathrm{g} / \mathrm{m}^{3}$ & Last 60 days & $-0.63(-2.24 ; 0.92)$ & $1.23(-0.21 ; 2.58)$ \\
& First 30 days & $-0.16(-0.38 ; 0.06)$ & $0.01(-0.38 ; 0.45)$ \\
& Last 30 days & $-0.15(-0.38 ; 0.06)$ & $0.01(-0.38 ; 0.45)$ \\
Normalized area of daily exposure over $15 \mu \mathrm{g} / \mathrm{m}^{3}$ & First 60 days & $-0.09(-0.40 ; 0.23)$ & $-0.03(-0.47 ; 0.37)$ \\
& Last 60 days & $-0.09(-0.39 ; 0.25)$ & $-0.03(-0.46 ; 0.39)$ \\
& First 30 days & $0.20(-3.28 ; 3.64)$ & $2.48(-4.72 ; 4.02)$ \\
& Last 30 days & $0.21(-3.07 ; 3.62)$ & $2.48(-4.74 ; 4.04)$ \\
& First 60 days & $-0.35(-4.78 ; 4.00)$ & $2.57(-4.75 ; 4.15)$ \\
& Last 60 days & $-0.34(-4.53 ; 3.99)$ & $2.58(-4.79 ; 4.14)$ \\
\hline
\end{tabular}


Table 2. RMSPE (Root Mean Square Predictive Error), MAPE (Mean Absolute Predictive Error), average length of the $95 \%$ predictive intervals (PI) and empirical coverage of the $95 \%$ predictive intervals obtained using the model that characterizes exposure using the third metric, that is, respectively, the "normalized area under the ambient exposure curve above $15 \mu \mathrm{g} / \mathrm{m}^{3}$ " and the "normalized area under the personal exposure curve above $15 \mu \mathrm{g} / \mathrm{m}^{3}$. The time window of exposure considered is the entire pregnancy. Each statistic is reported in grams

\begin{tabular}{|c|c|c|c|c|}
\hline & RMSPE & MAPE & $\begin{array}{c}\text { Average length } \\
\text { of } 95 \% \text { PI }\end{array}$ & $\begin{array}{c}\text { Empirical coverage } \\
\text { of } 95 \%\end{array}$ \\
\hline Ambient exposure (without spatial effects) & 428.52 & 334.71 & 1533.45 & $91.76 \%$ \\
\hline Ambient exposure (with spatial effects) & 424.98 & 331.57 & 1632.32 & $94.11 \%$ \\
\hline Personal exposure (with spatial effects) & 393.19 & 310.67 & 1666.09 & $97.74 \%$ \\
\hline
\end{tabular}

for personal exposure associates a moderate positive effect to those tracts, the model for ambient exposure associates to them a moderate negative effect.

The spatial map of the posterior standard deviation of the random spatial effects indicates that there is much more uncertainty in the estimates of these effects under the personal exposure scenario than under the ambient exposure scenario. In Figure 8 in the Appendix, lighter shades indicate smaller standard deviations, while darker shades indicate larger standard deviations. Such an increase in uncertainty under the personal exposure framework can be explained by the additional uncertainty in exposure provided by the distributions from the SHEDS-PM model and therefore is, arguably, more realistic than the uncertainty ascribed to the ambient exposure model.

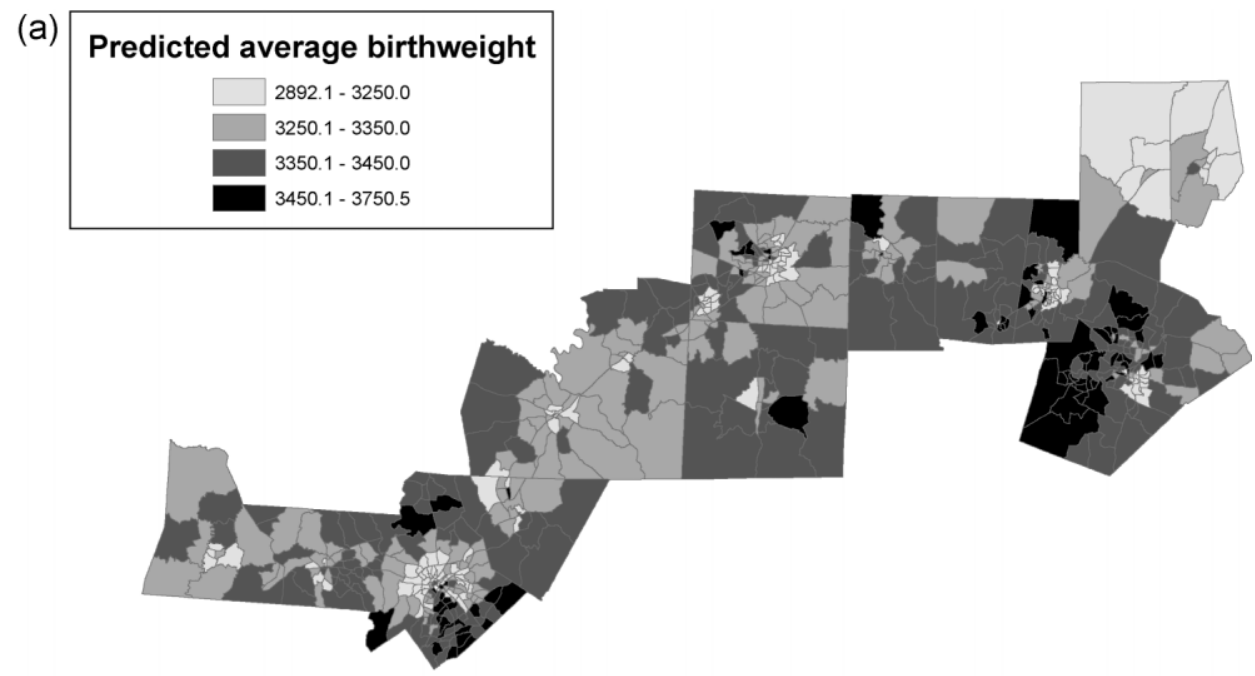

(b)

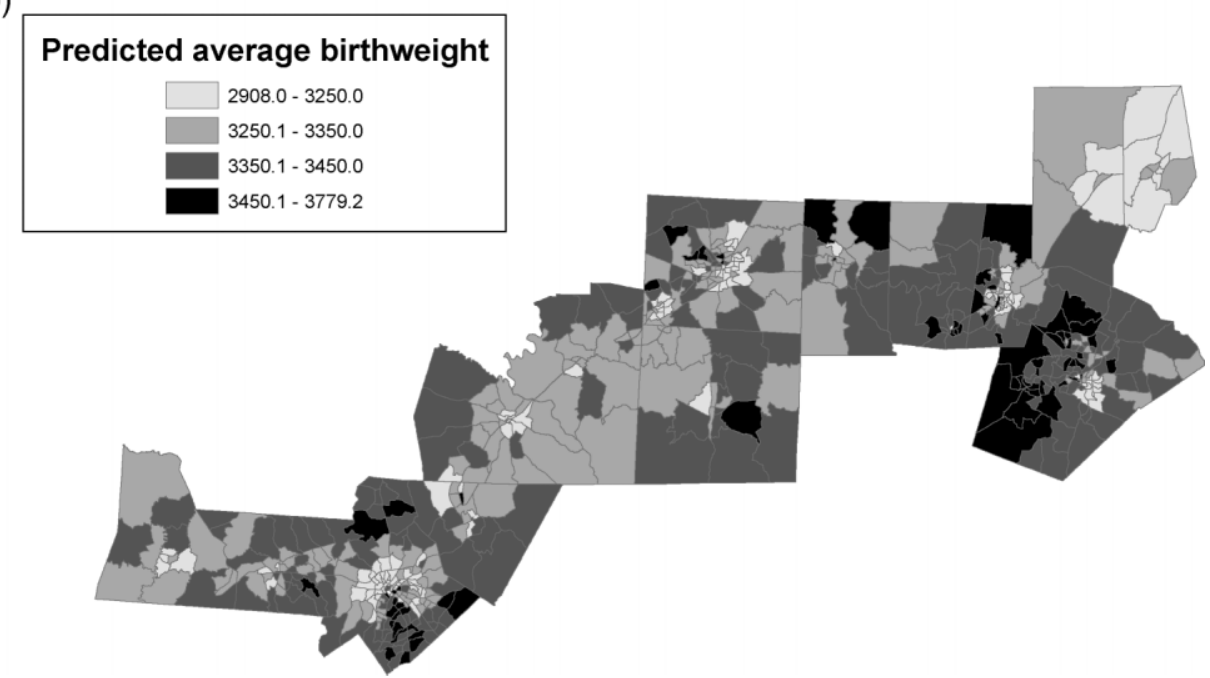

Figure 5. Posterior predictive mean of average birth weight (in grams) by census tract as obtained from the model where the exposure metric is: (a) "normalized area under the ambient exposure curve above $15 \mu \mathrm{g} / \mathrm{m}^{3}$ " versus (b) "normalized area under the personal exposure curve above $15 \mu \mathrm{g} / \mathrm{m}^{3}$ ". In both models the time window of exposure is the entire pregnancy. Additionally, both posterior predictive means are reported in grams 
To illustrate this last point, we have also assessed the in-sample predictive ability of our models by predicting birthweight for each mother in the dataset. Table 2 reports, in grams, the root mean square predictive error, the mean absolute predictive error, the average length of the $95 \%$ predictive interval and the empirical coverage of the $95 \%$ predictive interval for the models that use the normalized area under the ambient exposure curve, respectively, the personal exposure curve above $15 \mu \mathrm{g} / \mathrm{m}^{3}$ evaluated over the course of the entire pregnancy. To show how adding the spatial random effects improves the predictive performance of the model, in Table 2 we have also included results for a model that does not include spatial random effects. As the table shows, modeling the residual spatial variability does improve the predictive performance of the model. However, it is using personal exposure rather than ambient exposure that improves the results dramatically as the first model yields predictions that are on average closer to the observed values. Both models generate predictive intervals that have an empirical coverage very close to the nominal. In particular, the additional uncertainty in the personal exposure scenario is reflected in wider predictive intervals that yield an empirical coverage above the nominal. In both exposure scenarios, the predictive intervals are quite wide and this can be explained by the rather large residual standard deviation. After adjusting for socio-economic factors and maternal exposure, independently of the combination of exposure metric and time window of exposure used, the residual standard deviation of the models is estimated to be around 300-450 grams. Such large residual standard deviations are also obtained when no spatial random effects are included in the model.

We conclude this section by presenting spatial maps of the predicted average birthweight and the standard deviation of the predicted average birthweight by census tract. Comparing Figure 1(a) and Figure 5(a) and (b), we can clearly see that the model that uses the normalized area under the personal exposure curve above $15 \mu \mathrm{g} / \mathrm{m}^{3}$ reproduces the spatial pattern of average birthweight shown in Figure 1(a). On the other hand, the model that uses ambient exposure seems to underestimate average birthweight in some of the tracts, as the top right corner of the map indicates.

\section{SUMMARY AND DISCUSSION}

In this paper, we have presented a hierarchical modeling framework that illustrates how an exposure simulator can be used to relate a health outcome to personal exposure when both the health endpoint and personal exposure are modeled at the individual level. For our analysis, we have applied this modeling framework to birthweight as health endpoint and particulate matter as pollutant. However the model is general and we believe that this type of approach could be useful in determining long-term effects of exposure on other health outcomes given a window of exposure, for example, a year before the onset of a medical complication.

Differently from the findings of Mannes et al. (2005) and Bell et al. (2007), our analysis did not reveal any significant effect of maternal exposure to $\mathrm{PM}_{2.5}$ on birthweight at any time window of exposure. In particular, both Mannes et al. (2005) and Bell et al. (2007) have reported a significant adverse effect of exposure to $\mathrm{PM}_{2.5}$ during the second and third trimester of the pregnancy.

A comparison between our results and previous results cannot easily be made as our model estimates maternal ambient exposure not in terms of county-wide average ambient concentration (Bell et al., 2007a), city-wide average ambient concentration (Basu et al., 2004), or average concentration as reported by the closest monitors within a 5 mile radius from the mother's residence (Basu et al., 2004; Mannes et al., 2005). Additionally, as noted above, our study considers a spatial domain with lower concentration of fine particulate matter than the area considered by Mannes et al. (2005), who examines Sydney. Australia, and Bell et al. (2007), who study the relationship between maternal exposure to $\mathrm{PM}_{2.5}$ and birthweight in Connecticut and Massachusetts. Finally, our model includes spatial random effects for the census tract of maternal residence, that other studies have not adjusted for.

The spatial random effects play an important role in our model and we believe that they do affect the relationship between exposure and birthweight, as the estimates of exposure and birthweight change if the spatial random effects are not included in the model, even though they still remain non-significant. We believe that including these spatial random effects is appropriate as they account for the residual spatial variability in the response variable that is not accounted for by socio-economic factors and by exposure itself. Additionally, even though not in line with the findings of Mannes et al. (2005) and Bell et al. (2007) our results agree with those of Basu et al. (2004) and Parker et al. (2005) who, characterizing exposure using the average ambient concentration of $\mathrm{PM}_{2.5}$ measured by the closest monitoring sites to a mother's residence, didn't find any significant adverse effect of particulate matter on birthweight.

Our analysis has also revealed that models using personal exposure yield better predictions than models that use ambient exposure, thus arguing that personal exposure offers a better representation of an individual's exposure to particulate matter than ambient exposure.

For each subject in our dataset, we have characterized personal exposure using the SHEDS-PM simulator, by associating to each mother a distribution based on a set of 30 personal exposures for 30 individuals simulated within SHEDS-PM that match the selected mother in terms of age and census tract of residence. Since, among its output, SHEDS-PM also reports the contribution of ambient exposure on each simulated individual's personal exposure, it is possible to adapt the framework in this paper to assess the effect on birthweight of the contribution of a mother's exposure to particulate matter due to outdoor sources.

In our model, we have characterized each individual's ambient exposure during a particular time window of exposure using single values and not distributions. As we did under the personal exposure scenario, it is possible to associate to each individual a distribution for each combination of ambient exposure-time window of exposure. This can easily be achieved by using the method of McMillan et al. (2009), that we have employed to combine monitoring data with numerical model's gridded prediction of daily $\mathrm{PM}_{2.5}$ concentration. Instead of using the median of the predictive distribution yielded by the model of McMillan et al. (2009), we could use the entire distribution analogously to what is done in our model under the personal exposure scenario.

There are several ways in which our model can be extended: we have adjusted for gestational age using only a linear term. It would be possible to allow for a non-linear dependence of birth weight on gestational age, as Wang et al. (1997) have done. We have summarized the time series of both ambient and personal exposure using three metrics that highlight different aspects of an individual's exposure; other metrics could be developed and tested. Finally, we have predicted the ambient concentration of particulate matter at the census tract level by 
employing the fusion method of McMillan et al. (2009). While this certainly breaks with the previous practice of characterizing ambient exposure using monitoring data only, there are alternative ways to integrate the information contained in the monitoring data and in the numerical model daily prediction of $\mathrm{PM}_{2.5}$.

\section{DISCLAIMER}

The U.S. Environmental Protection Agency through its Office of Research and Development partially collaborated in the research described here. Although it has been reviewed by the Agency and approved for publication, it does not necessarily reflect the Agency's policies or views.

\section{Acknowledgements}

The authors thank Haluk Ozkaynak and Montserrat Fuentes for valuable discussions. This research was supported in part by USEPA Award No. RD-83329301-0 and by a National Research Council Research Associate Grant (No. 83323201-7) that supported Berrocal's postdoctoral fellowship at the US Environmental Protection Agency.

\section{REFERENCES}

Armstrong BK, White E, Saracci R. 1992. Principles of Exposure Measurement in Epidemiology. Oxford University Press: Oxford.

Banerjee S, Carlin BP, Gelfand AE. 2004. Hierarchical Modeling and Analysis for Spatial Data. Chapman \& Hall/CRC: Boca Raton, FL.

Basu R, Woodruff TJ, Parker JD, Saulnier L, Schoendorf KC. 2004. Comparing exposure metrics in the relationship between $\mathrm{PM}_{2.5}$ and birth weight in California. Journal of Exposure Analysis and Environmental Epidemiology 14: 391-396.

Bell ML, Ebisu K, Belanger K. 2007. Ambient air pollution and low birth weight in Connecticut and Massachussetts. Environmental Health Perspectives 115: $1118-1124$

Besag J. 1974. Spatial interaction and the statistical analysis of lattice systems. Journal of the Royal Statistical Society Series B 36: $192-236$.

Besag J, Green PJ, Higdon D, Mengersen K. 1995. Bayesian computation and stochastic systems. Statistical Science 10: 3-66.

Bobak M. 2000. Outdoor air pollution, low birth weight, and prematurity. Environmental Health Perspectives 108: 173-176.

Bobak M, Leon DA. 1992. Air pollution and infant mortality in the Czech Republic, 1986-1988. Lancet 340: 1010-1014.

Braga ALF, Zanobetti A, Schwartz J. 2001. The lag structure between particulate air pollution and respiratory and cardiovascular deaths in ten US cities. Journal of Occupational and Environmental Medicine 43: 927-933.

Burke JM, Zufall MJ, Özkaynak H. 2001. A population exposure model for particulate matter: case study results for PM 2.5 in Philadelphia, PA. Journal of Exposure Analysis and Environmental Epidemiology 11: 470-489.

Calder CA, Holloman CH, Bortnick SM, Strauss WJ, Morara M. 2008. Relating ambient particulate matter concentration levels to mortality using an exposure simulator. Journal of the American Statistical Association 103: 137-148.

Chen L, Yang W, Jennison BJ, Goodrich A, Omaye ST. 2002. Air pollution and birth weight in Northern Nevada, 1991-1999. Inhalation Toxicology 14: $141-157$.

Cressie NAC. 1993. Statistics for Spatial Data. Wiley: New York.

Dockery DW, Pope CAI. 1994. Acute respiratory effects of particulate air pollution. Annual Review of Public Health 15: 107-132.

Dockery DW, Spengler J. 1981. Personal exposure to respirable particulates and sulfates. Journal of air pollution control association 31: 153-159.

Dockery DW, Pope CA3rd, Xu X, Spengler JD, Ware JH, Fay ME, Ferris BG Jr,, Speizer FE. 1993. An association between air pollution and mortality in six US cities. New England Journal of Medicine 329: 1753-1759.

Dominici F, Zeger SL, Samet JM. 2000. A measurement error model for time-series studies of air pollution and mortality. Biostatistics 1: 157-175.

Dugandzic R, Dodds L, Stieb D, Smith-Doiron M. 2006. The association between low level exposures to ambient air pollution and term low birth weight: a retrospective cohort study. Environmental Health 5: 18-25.

Fuentes M, Song H-R, Ghosh SK, Holland DM, Davis JM. 2006. Spatial association between speciated fine particles and mortality. Biometrics 62: 855-863. Gelfand AE, Smith AFM. 1990. Sampling-based approaches to calculating marginal densities. Journal of the American Statistical Association 85: 398-409.

Gelfand AE, Sahu SK, Carlin BP. 1996. Efficient parametrization for generalized linear mixed models (with discussion). In Bayesian Statistics Vol. 5, JM, Bernardo JO, Berger AP, D AFM Smith (eds). Oxford University Press, Oxford: 165-180.

Goldberg MS, Burnett RT, Bailar JC 3rd, Brook J, Bonvalot Y, Tamblyn R, Singh R, Valois MF, Vincent R. 2001. The association between daily mortality and ambient air particle pollution in Montreal. Environmental Research 86(1): 26-36.

Gouveia N, Bremner SA, Novaes HMD. 2004. Association between ambient air pollution and birth weight in Sao Paulo, Brazil. Journal of Epidemiology and Community Health 58: 11-17.

Gryparis A, Paciorek CJ, Zeka A, Schwartz J, Coull BA. 2009. Measurement error caused by spatial misalignment in environmental epidemiology. Biostatistics 10: $258-274$.

Ha E-H, Hong Y-C, Lee B-E, Woo B-H, Schwarz J, Christiani DC. 2001. Is air pollution a risk factor for low birth weight in Seoul? Epidemiology 12: 643-648.

Hansen C, Neller A, Williams G, Simpson R. 2006. Maternal exposure to low levels of ambient air pollution and preterm birth in Brisbane, Australia. Epidemiology 935-941.

Heinrich J, Hoelscher B, Wjst M, Ritz B, Cyrys J, Wichmann H. 1999. Respiratory diseases and allergies in two polluted areas in East Germany. Environmental Health Perspectives 107: 53-62.

Hoek G, Brunekreef B, Fisher P, Wijnen JV. 2001. The association between air pollution and heart failure, arrythmia, embolism, thromobosis, and other cardiovascular causes of death in a time series study. Epidemiology 12: 355-357.

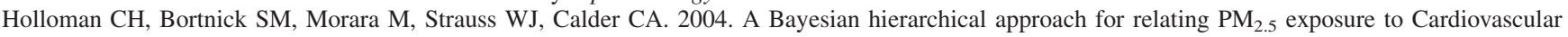
mortality in North Carolina. Environmental Health Perspectives 112: 1282-1288.

Katsouyanni K, Touloumi G, Samoli E, Gryparis A, Le Tertre A, 2001. Confounding and effect modification in the short-term effects of ambient particles on total mortality: results from 29 European cities within the APHEA2 project. Epidemiology 12: 521-531.

Law PL, Lioy PJ, Zelenka MP, huber AH, McCurdy TR. 1997. Evaluation of a probabilistic exposure model applied to carbon monoxide (pNEM/CO) using Denver personal exposure monitoring data. Journal of the Air and Waste Management Association 47: 491-500.

Lee BE, Ha EH, Park HS, Kim YJ, Hong YC, Kim H, Lee JT. 2003. Exposure to air pollution during different gestational phases contributes to risks of low birth weight. Human Reproduction 18: 638-643. 
Lin C-M, Li C-Y, Yang G-Y, Mao I-F. 2004. Association between maternal exposure to elevated ambient sulfure dioxide during pregnancy and term low birth weight. Environmental Research 96: 41-50.

Lioy PJ, Waldman BT, Butler J, Piertarinen C. 1990. The personal, indoor, and outdoor concentration of $\mathrm{PM}_{10}$ measured in an industrial community during the winter. Atmospheric Environment 24: 57-66.

Liu S, Krewski D, Shi Y, Chen Y, Burnett RT. 2003. Association between gaseous ambient air pollutants and adverse pregnancy outcomes in Vancouver, Canada. Environmental Health Perspectives 111: 1773-1778.

Maisonet M, Bush T, Correa A, Jaakkola JJK. 2001. Relation between ambient air pollution and low birth weight in the Northeastern United States. Environmental Health Perspectives 109(S3): 351-356.

Maisonet M, Correa A, Misra D, Jaakkola JJK. 2004. A review of the literature on the effects of ambient air pollution on air growth. Environmental Research 95: 106-115.

Mannes T, Jalaludin B, Morgan G, Lincoln D, Sheppeard V, Corbett S. 2005. Impact of ambient air pollution on birth weight in Sydney, Australia. Occupational and Environmental Medicine 62: 524-530.

McBride SJ, Williams RW, Creason J. 2007. Bayesian hierarchical modeling of personal exposure to particulate matter. Atmospheric Environment 41: 6143-6155.

McMillan N, Holland DM, Morara M, Feng J. 2010. Combining numerical model output and particulate data using Bayesian space-time modeling. Environmetrics 21: 41-65.

Ostro BD, Broadwin R, Lipsett MJ. 2000. Coarse and fine particles and daily mortality in the Coachella Valley: a follow-up study. Journal of Exposure Analysis and Environmental Epidemiology 10: 412-419.

Özkaynak H, Sue J, Spengler J, Wallace L, Pellizzari E, Jenkins P. 1996. Personal exposure to airborne particles and metals: results from the particle team study in Riverside, California. Journal of Exposure Analysis and Environmental Epidemiology 6: 57-78.

Ozone 2006. Air Quality Criteria for Ozone and Related Photochemical Oxidants (Final). US Environmental Protection Agency: Washington, DC; EPA/600/ $\mathrm{R}-05 / 004 \mathrm{aF}-\mathrm{cF}$.

Parker JD, Woodruff TJ, Basu R, Schoendorf KC. 2005. Air pollution and birth weight among term infants in California. Pediatrics 115: 121-128.

Pope CA, Dockery DW, Schwartz J. 1995. Review of epidemiological evidence of health-effects of particulate air-pollution. Inhalation Toxicology 7: 1-18.

Reich BJ, Fuentes M, Burke J. 2009. Analysis of the effects of ultrafine particulate matter while accounting for human exposure. Environmetrics 20: 131-146.

Ritz B, Yu F. 1999. Effect of ambient carbon monoxide on low birth weight among children born in Southern California between 1989 and 1993. Environmental Health Perspectives 107: 17-25.

Ritz B, Yu F, Chapa G, Fruin S. 2000. Effect of air pollution on preterm birth among children born in Southern California between 1989 and 1993. Epidemiology 5: 502-511.

Rogers JF, Dunlop AL. 2000. Association of very low birth weight with exposures to environmental sulfur dioxide and total suspended particulates. American Journal of Epidemiology 151: 602-612.

Rogers JF, Dunlop AL. 2005. Air pollution and very low birth weight infants: a target population. Pediatrics 156-165.

Saldiva PHN, Pope CA 3rd, Schwartz J, Dockery DW, Lichtenfels AJF, et al. 1995. Air pollution and mortality in elderly people: a time series study in Sã0 Paulo, Brazil. Archives of Environmental Health 50: 159-163.

Samet JM, Zeger SL, Dominici F, Curriero F, Coursac I, Dockery DW. 2000. The national morbidity, mortality and air pollution study. Part I: methods and methodologic issues. Part II: morbidity, mortality, and air pollution in the United States, Research Report No. 9, Health Effects Institute: Cambridge, MA.

Schwartz J. 1994. Air pollution and daily mortality: a review and meta analysis. Environmental Research 64: 36-52.

Schwartz J. 1999. Air pollution and hospital admissions for heart diseases in eight U.S. counties. Epidemiology 10: 17-22.

Schwartz J, Dockery DW, Neas LM, Wypij D, Ware JH, et al., J.D.S., 1994. Acute effects of summer air pollution on respiratory symptom reporting in children. American Journal of Respiratory Critical Care Medicine 150: 1234-1242.

Shaddick G, Lee D, Zidek JV, Salway R. 2008. Estimating exposure response functions using ambient pollution concentration. Annals of Applied Statistics 2: $1249-1270$.

Spengler JD, Treitman RD, Tosteson TD, Mage DT, Soczek ML. 1985. Personal exposure to respirable particulates and implications for air pollution epidemiology. Environmental Science Technology 19: 700-707.

Šrám RJ. 1999. Impact of air pollution on reproductive health. Environmental Health Perspectives 107: 542-543.

Šrám RJ, Blinková B, Dejmek J, Bobak M. 2005. Ambient air pollution and pregnancy outcomes: a review of the literature. Environmental Health Perspectives 113: $375-382$.

Wallace L. 1996. Indoor particles: a review. Journal of the Air and Waste Management Association 46: 98-126.

Wang X, Ding H, Ryan L, Xu X. 1997. Association between air pollution and low birth weight: a community based study. Environmental Health Perspectives 105: 514-521.

Woodruff TJ, Grillo J, Schoendorf KC. 1997. The relationship between selected causes of postnatal infant mortality and particulate air pollution in the United States. Environmental Health Perspectives 105: 608-612.

Yang CY, Tseng YT, Chang CC. 2003. Effects of air pollution on birth weight among children born between 1995 and 1997 in Kaohsiung, Taiwan. Journal of Toxicology and Environmental Health 66: 807-816.

Zanobetti A, Schwartz J, Dockery DW. 2000. Airborne particles area risk factor for hospital admissions for heart and lung disease. Environmental Health Perspectives 108: 1071-1077.

Zeger S, Thomas D, Dominici F, Cohen A, Schwartz J, Dockery D, Samet J. 2000. Measurement error in time-series studies of air pollution: concepts and consequences. Environmental Health Perspectives 108: 419-426.

Zidek JV, Meloche J, Chatfield C, White R. 2003. A computational model for estimating personal exposure to air pollutants with application to London's PM10 in 1997. Technical Report of the Statistical and Applied Mathematical Sciences Institute.

Zidek JV, Shaddick G, Meloche J, Chatfield C, White R. 2007. A framework for predicting personal exposures to environmental hazards. Environmental and Ecological Statistics 14: 411-431. 


\section{APPENDIX}
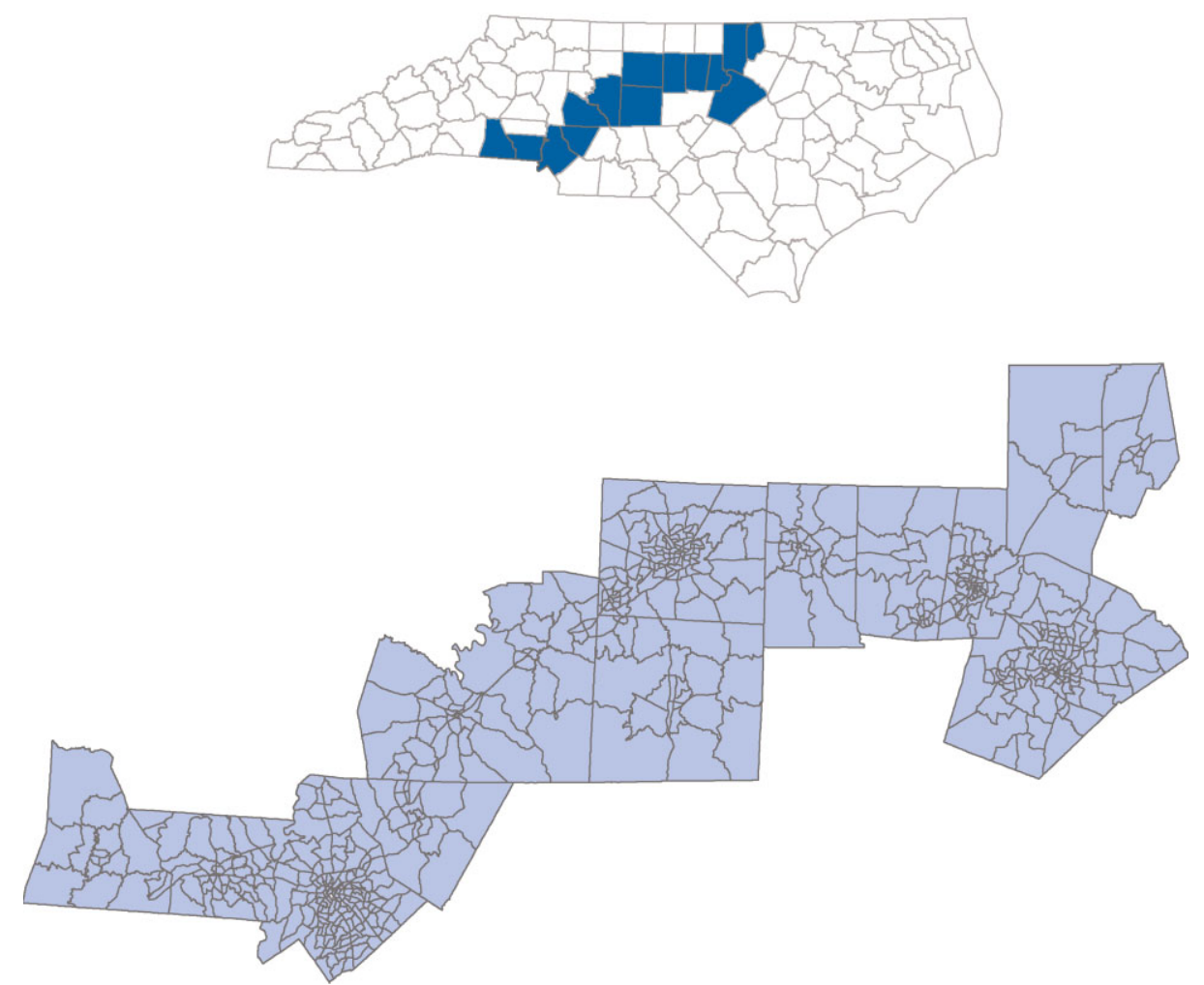

Figure 6. Fourteen counties in North Carolina included in this analysis (top panel) and, in detail, the census tracts contained in the 14 counties (bottom panel). This figure is available in color online at wileyonlinelibrary.com/journal/environmetrics 
Environmetrics

V. J. BERROCAL ET AL.

(a)
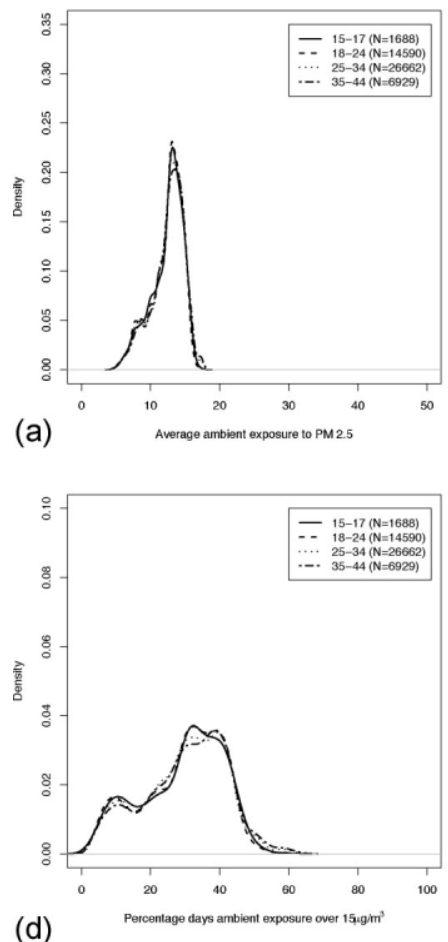

(d)

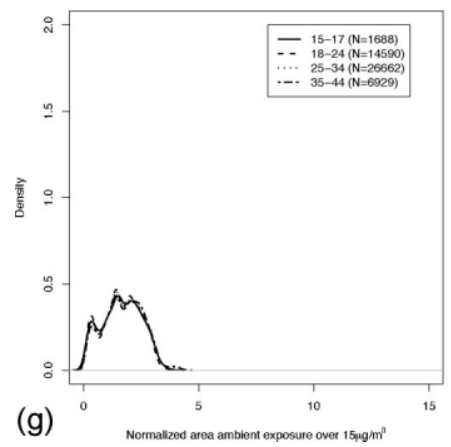

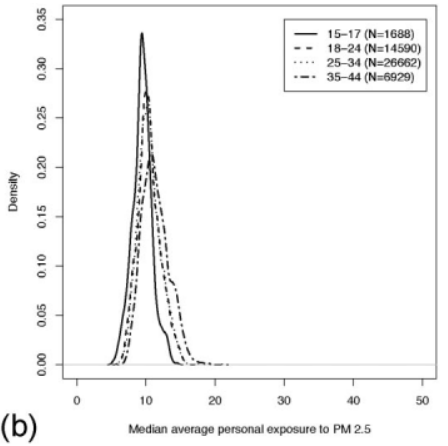

(b)

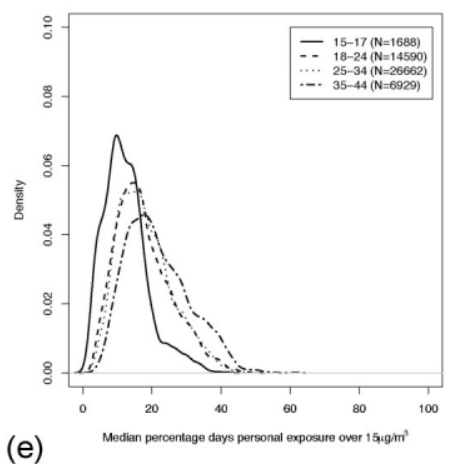

(e)

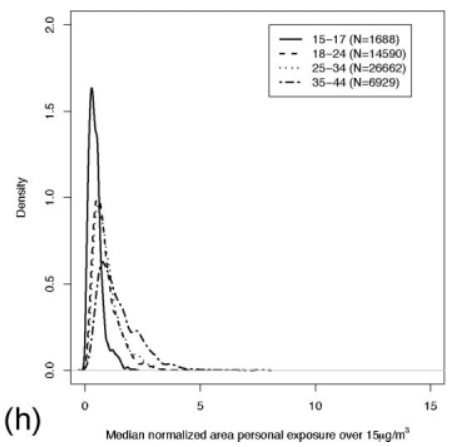

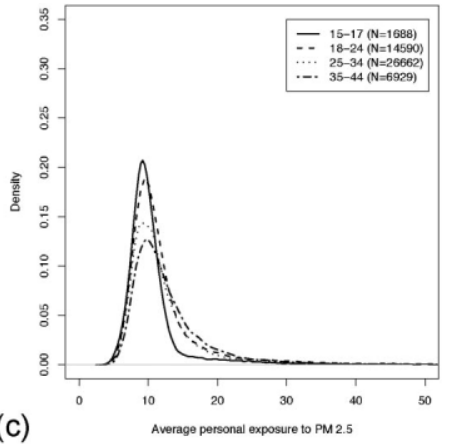

(c)

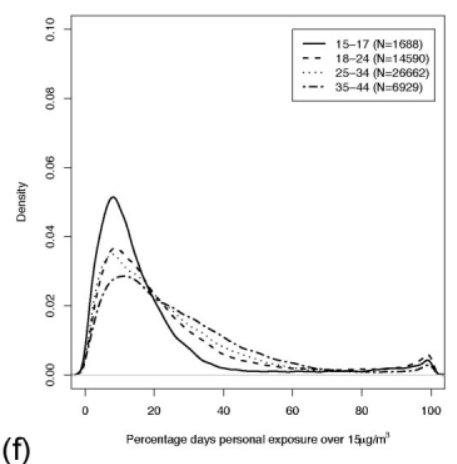

(f)

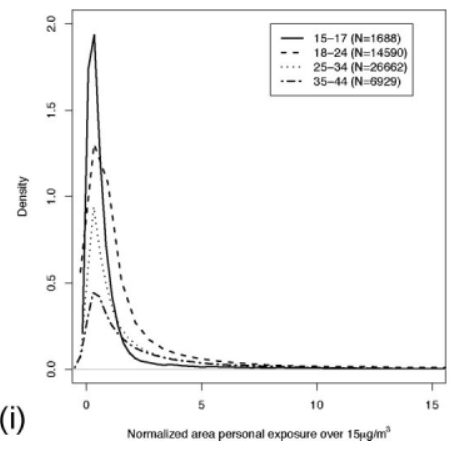

Figure 7. Kernel density estimates of the distributions of the three metrics considered over the entire pregnancy: (a) Average ambient exposure, (b) median average personal exposure, and (c) average personal exposure, (d) percentage of days ambient exposure exceeded $15 \mu \mathrm{g} / \mathrm{m}^{3}$, (e) median percentage of days personal exposure exceeded $15 \mu \mathrm{g} / \mathrm{m}^{3}$, (f) percentage of days personal exposure exceeded $15 \mu \mathrm{g} / \mathrm{m}^{3}$, (g) normalized area under ambient exposure curve above $15 \mu \mathrm{g} / \mathrm{m}^{3}$, (h) median normalized area under personal exposure curve above $15 \mu \mathrm{g} / \mathrm{m}^{3}$, and (i) normalized area under personal exposure curve above 15 $\mu \mathrm{g} / \mathrm{m}^{3}$. In each panel, the kernel density estimates are grouped by age groups

wileyonlinelibrary.com/journal/environmetrics

Copyright (C) 2011 John Wiley \& Sons, Ltd.

Environmetrics 2011; 22: 553-571 
(a)
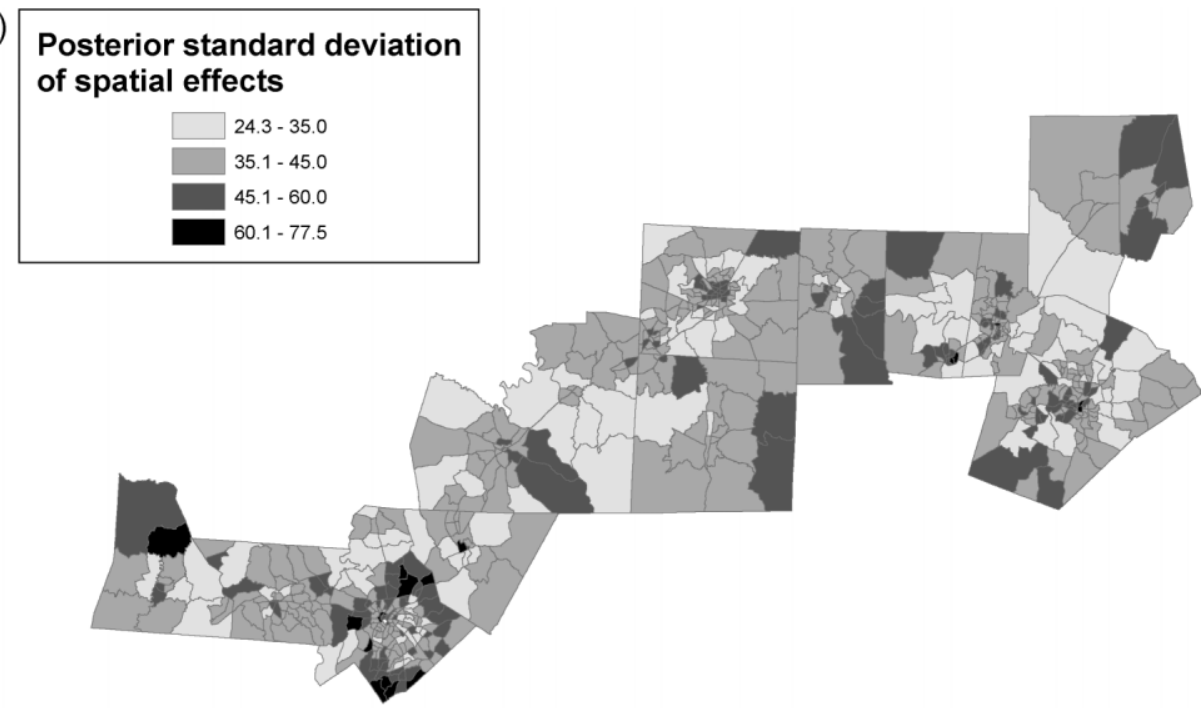

(b)
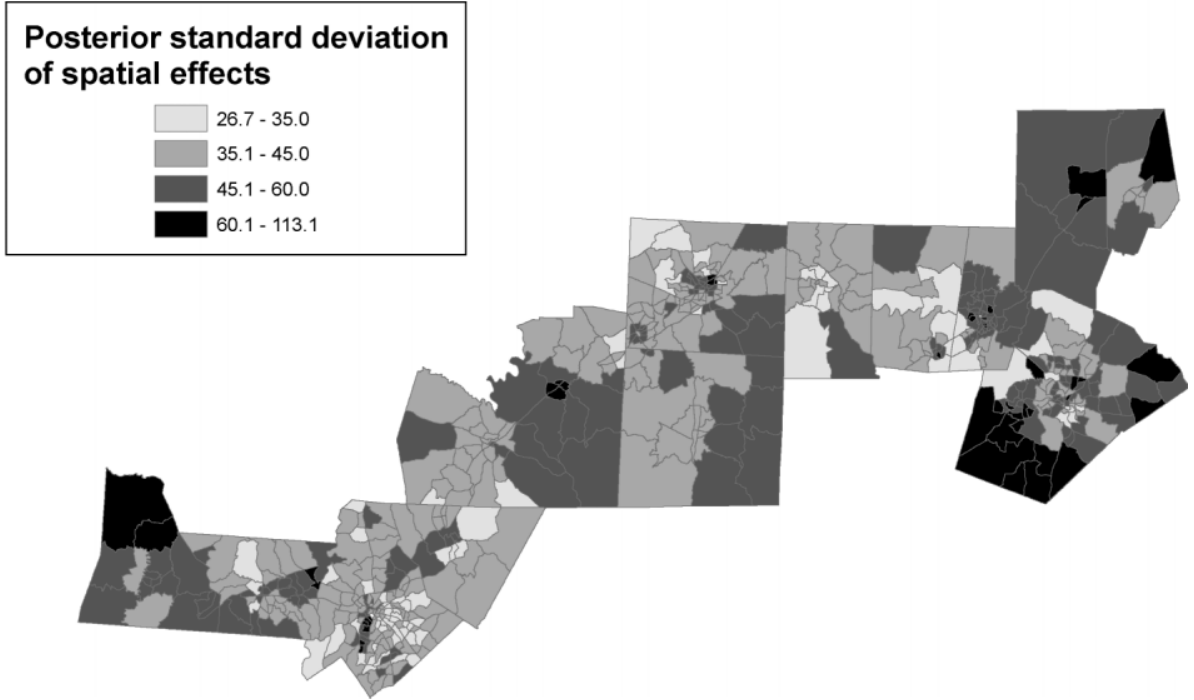

Figure 8. Posterior standard deviations of the random spatial effects obtained from the models where the exposure metric is: (a) "normalized area under the ambient exposure curve above $15 \mu \mathrm{g} / \mathrm{m}^{3}$ " and (b) "normalized area under the personal exposure curve above $15 \mu \mathrm{g} / \mathrm{m}^{3}$ ". In both models the time window of exposure is the entire pregnancy. Additionally, both posterior standard deviations are reported in grams 